\title{
Segregación escolar público-privada en España
}

\author{
F. Javier Murillo \\ Guillermina Belavi \\ Lina M. Pinilla \\ Universidad Autónoma de Madrid \\ javier.murillo@uam.es; guillermina.belavi@uam.es; linamarcelapinilla@gmail.com
}

Recepción: 05-12-2016

Aceptación: 05-09-2017

Publicación: 16-05-2018

\section{Resumen}

Hace más de 30 años se reguló en España la doble red de centros públicos y privados. Es buen momento para preguntarse qué ha significado en términos de equidad. Este artículo aborda la aportación diferencial de la educación pública y privada a la segregación escolar de carácter socioeconómico. Más concretamente, tiene como objetivos, por un lado, estimar la magnitud de la segregación por nivel socioeconómico de los centros de Educación Secundaria Obligatoria de titularidad pública y de titularidad privada y, por otro, determinar la contribución diferencial de ambos a la segregación global en cada comunidad autónoma. Para ello, se realiza una explotación secundaria de los datos PISA-2015 y se estima el índice de segregación de raíz cuadrada de Hutchens y su descomposición en los subsistemas de educación pública y privada para cuatro colectivos de estudiantes: el $10 \%$ y el $25 \%$ de los estudiantes con familias con menor nivel socioeconómico, y el 10\% y 25\% de los de mayor nivel. Los resultados apuntan, en primer lugar, a una gran variabilidad en la segregación escolar entre comunidades, tanto en magnitud como en composición público-privada. En segundo término, se encuentra una notable segregación de los centros privados a los estudiantes de familias con menor nivel socioeconómico y cultural y de los centros públicos a los de mayor nivel, lo que apunta a la importante contribución que la educación privada realiza a la segregación escolar global por carácter socioeconómico.

Palabras clave: segregación escolar; titularidad de centro; nivel socioeconómico; educación secundaria; PISA; España 


\title{
Abstract. Public-private school segregation in Spain
}

More than 30 years ago, the double network of private and public schools was regulated in Spain. Today is time to inquire into what this has meant in terms of equity. This article addresses the differential contribution of public and private education to socioeconomic school segregation. It aims to estimate the magnitude of segregation by socioeconomic status in public and private compulsory secondary education schools and determine the contribution of both to global segregation in autonomous communities. For this purpose, a secondary analysis of PISA-2015 data was performed and the Hutchens square root segregation index and its decomposition in the public and private education subsystems were estimated for four groups of students: $10 \%$ and $25 \%$ of students from families with a lower socioeconomic status and $10 \%$ and $25 \%$ of students from families with a higher socioeconomic status. The results reveal a high variability in school segregation between communities in terms of both magnitude and public-private distribution. Secondly, a significant segregation of students with lower socioeconomic and cultural status at private schools and students with higher socioeconomic and cultural status at public schools was found. The results reveal the important contribution of private schools to global segregation by socioeconomic status.

Keywords: school segregation; school partnership; socioeconomic status; secondary education; PISA; Spain

\author{
Sumario \\ 1. Introducción 4. Resultados \\ 2. Marco teórico 5. Conclusiones \\ 3. Método Referencias bibliográficas
}

\section{Introducción}

Hace más de 30 años que se reguló en España la doble red de centros públicos y de centros privados financiados con fondos públicos. En ese tiempo no ha parado de aumentar el peso de la educación privada en detrimento de la pública, y no hay muestras de que esa tendencia vaya a revertir. Tres décadas es un tiempo razonable para evaluar qué ha significado en términos de equidad.

Uno de los indicadores de equidad educativa es la llamada segregación escolar. Este término hace referencia a la distribución desigual de los estudiantes en los centros educativos según sus características personales o sociales; por ejemplo, el país de nacimiento, la pertenencia a un grupo étnico-cultural, la capacidad o el nivel socioeconómico y cultural de las familias (Allen y Vignoles, 2007; Frankel y Volij, 2011). Dentro de ellas destaca especialmente esta última, la segregación escolar por nivel socioeconómico, dada su especial incidencia en las desigualdades sociales (Orfield y Lee, 2005).

¿Cuál es la magnitud de la segregación por nivel socioeconómico de las escuelas públicas?, ¿y de las privadas? ¿Cuánto aporta cada una a la segregación escolar por nivel socioeconómico en Educación Secundaria Obligatoria en 
cada comunidad autónoma española? Las respuestas nos permitirán visibilizar la contribución de la educación privada a la equidad del sistema educativo español.

\section{Marco teórico}

\subsection{La educación público-privada}

¿La educación privada es beneficiosa o perjudicial para el sistema educativo? La respuesta y el grado de consenso que genera varían, fundamentalmente, en función del criterio de consideración. En términos de excelencia, por ejemplo, existen investigaciones con resultados encontrados. Quienes apuestan por la escolarización privada aluden a la conocida hipótesis Coleman-Hoffer (Coleman y Hoffer, 1987; Corten y Dronkers, 2006), que sostiene que el rendimiento de los estudiantes es superior en las escuelas privadas que en las públicas. Estos resultados han sido ampliamente discutidos por la comunidad científica refiriéndose, entre otras razones, a las limitaciones metodológicas del estudio (Portes, 1998; Sandefur y Laumann, 1998; Morgan y Sørensen, 1999). Algunos estudios posteriores han encontrado que los efectos positivos de la escolaridad en centros privados son muy limitados (Angrist et al., 2002; Stevans y Sessions, 2000); otros han aportado evidencias empíricas de la ausencia de relación: en Estados Unidos (Dronkers, 2004; Dronkers y Robert, 2008; Goldhaber, 1996), en Reino Unido (Smith y Naylor, 2005), en Alemania (Fertig, 2003) o en Italia (Abburrá, 2005), por poner algunos ejemplos. Incluso hay quienes señalan que los resultados académicos de los estudiantes en los centros privados son peores que los que se obtienen en la escuela pública (Barbetta y Turati, 2003). Pero también hay evidencias de una eficacia diferencial. Tokman (2002), por ejemplo, encontró que las escuelas públicas son más eficaces para los estudiantes de familias de menor nivel socioeconómico.

El grado de coincidencia entre los resultados de las investigaciones sobre la educación privada frente a la pública es mayor cuando se consideran otros criterios. En términos de equidad, el debate se focaliza en determinar si la enseñanza privada contribuye a una mejora de los sistemas educativos o si, por el contrario, incrementa las desigualdades sociales. En este caso, hay más acuerdo en considerar que los criterios de acceso y selección de la escuela privada no facilitan la equidad (Arcidiácono et al., 2014; Betts y Fairlie, 2014; Rumberger y Parlardy, 2005). Por ejemplo, los resultados del canónico estudio realizado por Coleman, Hoffer y Kilgore (1982) apuntan a que el nivel socioeconómico de los estudiantes es hasta tres veces más alto en las escuelas privadas que en las públicas, provocando poco contacto entre estudiantes de diferente nivel socioeconómico. Según los autores, la proporción de estudiantes con alto nivel socioeconómico cuyos compañeros de clase tienen bajo nivel socioeconómico es de 0,14 en las escuelas públicas y de 0,07 en las privadas. De esta forma, la investigación es clara al señalar a la enseñanza pública como promotora de la equidad social entre los estudiantes, dado que asegura que las condiciones pre- 
vias de los estudiantes no se conviertan en barreras para su desarrollo (Elacqua et al., 2016; OCDE, 2009).

Estos resultados pueden ser inquietantes para el caso español (Fernández Enguita, 2008), pues el porcentaje de estudiantes matriculados en escuelas de titularidad privada en España es importante, sobre todo si se contrasta con países de su entorno. En el año 2014 el 85,2\% de los estudiantes de Educación Secundaria Obligatoria de la Unión Europea estudiaban en centros públicos (Eurostat, 2016). En España esa cifra era del 65,6\% de los estudiantes, lo que la ubica como el cuarto país de Europa (y de los países de la OCDE) con un menor porcentaje de estudiantes matriculados en escuelas de titularidad pública, solo detrás de Reino Unido (43,1\%), Bélgica (42,0\%) y Malta $(53,8 \%)$.

Frente a esta situación global, es preciso tener en cuenta que la cifra es producto de una gran variabilidad de porcentajes, pues en España la distribución público-privada es muy diferente entre comunidades autónomas. En ese mismo nivel de ESO y con datos del curso 2014/15 (MEC, 2016), por ejemplo, el 46,4\% de los estudiantes del País Vasco asistían a centros públicos; el 52,0\% de la Comunidad de Madrid; el 80,1\% de los estudiantes de Castilla-La Mancha, y el 76,7\% de los estudiantes de Extremadura (tabla 1). Además, la evolución es clara: el peso de la educación privada ha crecido en estos años en el conjunto del Estado, así como en la mayoría de las comunidades, aunque su incremento en los últimos años parece haberse ralentizado (tabla 1).

España es un país especialmente interesante a la hora de considerar los efectos de la educación privada en la segregación escolar, tanto por su peso creciente a nivel nacional como por la heterogeneidad de casos que presenta en su interior cuando se considera la situación particular de las comunidades. Existen estudios regionales que permiten profundizar en las condiciones de heterogeneidad de la segregación escolar en las distintas comunidades, entre ellos, cabe destacar investigaciones llevadas adelante en Cataluña (Benito y González, 2007; Bonal, 2012), en la Comunidad de Madrid (García Fernández y Moreno, 2002; Poveda et al., 2007) y en Andalucía (García Castaño y Rubio Gómez, 2013).

La segregación escolar público-privada debe ser analizada a la luz de las características del sistema educativo español, pues el avance de la educación privada fue diferente según el contexto histórico y sociopolítico de los países o regiones en que desarrollaba. En el sistema español, la educación privada se rige por una política de financiación mediante fondos públicos, lo que se conoce como educación concertada. Esta estrategia de financiación pública fue repetidamente adoptada por países y regiones en los que el sector privado ha tenido una presencia histórica significativa como proveedor de educación; además de España, son buenos ejemplos Holanda, Bélgica o Irlanda (Alegre Canosa, 2010). De esta manera, los centros privados reciben partidas presupuestarias públicas a condición, entre otras cosas, de adecuar su oferta formativa a los currículums oficiales estandarizados, no aplicar tasas de matriculación a sus estudiantes y aceptar la introducción de determinados criterios comunes, establecidos públicamente, en el proceso de admisión del alumnado. 
Tabla 1. Evolución del porcentaje de estudiantes de Educación Secundaria Obligatoria en centros públicos por comunidad autónoma

\begin{tabular}{lccccc}
\hline & $1994 / 95$ & $1999 / 00$ & $2004 / 05$ & $2009 / 10$ & $2014 / 15$ \\
\hline Andalucía & 80,9 & 75,0 & 75,2 & 75,2 & 75,2 \\
Aragón & 81,6 & 59,5 & 61,8 & 64,2 & 65,8 \\
Principado de Asturias & 93,7 & 68,9 & 65,1 & 65,1 & 66,3 \\
Islas Baleares & 91,0 & 57,6 & 59,2 & 61,0 & 60,8 \\
Canarias & 100,0 & 77,9 & 76,7 & 75,4 & 75,8 \\
Cantabria & 84,5 & 64,1 & 63,0 & 62,5 & 66,3 \\
Castilla y León & 84,9 & 66,3 & 64,4 & 63,4 & 64,0 \\
Castilla-La Mancha & 90,1 & 79,4 & 80,4 & 80,8 & 80,1 \\
Cataluña & 74,7 & 55,5 & 58,1 & 60,2 & 62,0 \\
Extremadura & 90,5 & 78,6 & 77,6 & 77,1 & 76,7 \\
Galicia & 92,3 & 73,3 & 70,9 & 69,2 & 70,3 \\
La Rioja & 75,9 & 61,7 & 63,6 & 64,2 & 61,4 \\
Comunidad de Madrid & 83,0 & 56,6 & 54,4 & 51,6 & 52,0 \\
Región de Murcia & 86,9 & 74,3 & 72,7 & 71,1 & 69,8 \\
C. Foral de Navarra &. & 57,5 & 59,7 & 60,9 & 60,2 \\
País Vasco &. & 42,7 & 42,1 & 45,1 & 46,4 \\
Comunidad Valenciana & 88,8 & 66,6 & 67,2 & 66,1 & 63,8 \\
Ceuta & 100,0 & 77,2 & 70,8 & 72,6 & 73,6 \\
Melilla & 79,5 & 86,5 & 88,3 & 89,5 & 89,2 \\
Total/promedio & 84,6 & 66,4 & 66,3 & 65,8 & 65,8 \\
\hline
\end{tabular}

Fuente: estadística de las enseñanzas no universitarias. Subdirección General de Estadística y Estudios del Ministerio de Educación, Cultura y Deporte.

El sistema educativo español adoptó estas pautas de privatización de manera muy generalizada, pues solo un pequeño porcentaje de establecimientos educativos se financia de forma exclusivamente privada. Para la educación secundaria, este porcentaje alcanza apenas el 3,53\% de los centros españoles, de manera que la opción de la escuela privada no concertada, sin condicionamientos, pero también sin financiamiento público, no ha calado en España. Por ello, el análisis de los efectos de la educación privada en materia de segregación debe realizarse sobre el trasfondo de la normativa bajo cuyo condicionamiento reciben financiación pública las escuelas concertadas, especialmente en lo que atañe a la libertad de elección de centro y al acceso en condiciones de igualdad.

En España, estos condicionamientos se rigen por el artículo 84 de la Ley Orgánica 8/2013, del 9 de diciembre, para la mejora de la calidad educativa (LOMCE), artículo que se mantiene de la anterior Ley Orgánica 2/2006, del 3 de mayo, de Educación. Este marco ordena la admisión de los estudiantes en centros financiados con fondos públicos (tanto públicos como privados) y establece la normativa que obliga a las administraciones educativas a garantizar el derecho a la educación, el acceso en condiciones de igualdad y la libertad de elección de centro. Según la letra de la ley, todos los centros sostenidos con fondos públicos deben atender a una escolarización equilibrada del alumnado 
con necesidades específicas de apoyo educativo. De forma genérica, el artículo establece también los requisitos para casos en los que no existan plazas suficientes para la admisión de alumnos en los centros. En estos casos, requiere considerar la existencia de hermanos matriculados en el centro; padres, madres o tutores legales que trabajen en el mismo; proximidad del domicilio o del lugar de trabajo de alguno de sus padres, madres o tutores legales; renta per cápita de la unidad familiar; familia numerosa; situación de acogimiento familiar del alumno o la alumna y concurrencia de discapacidad en el alumno o la alumna o en alguno de sus padres, madres o hermanos.

Si la iniciativa privada-concertada influye en la segmentación de la educación, estaríamos por lo tanto frente un problema de incumplimiento de la ley. Como señaló Bonal (2002: 27) hace 14 años, pero con más vigencia en la actualidad que nunca:

La normativa referida a la política de admisión de alumnos y a la garantía de la gratuidad de la enseñanza no se ha aplicado con rigor, de tal modo que la enseñanza privada concertada no ofrece las mismas condiciones de escolarización que los centros públicos: persisten prácticas de selección de alumnos y se cobran cuotas en los niveles obligatorios del sistema educativo.

Esto genera que familias con mayores niveles socioeconómicos y culturales escojan determinados centros para escolarizar a sus hijos e hijas, mientras que padres y madres con menos recursos no disponen de la posibilidad real de elegir la escuela deseada. Esta imposibilidad de elegir el centro de escolarización con la libertad que la ley proclama, a la postre, genera situaciones de segregación escolar por nivel socioeconómico que desembocan en una falta de real igualdad de oportunidades.

Dentro de ese debate, cobra especial importancia el estudio de los efectos del peso de la educación privada en la segregación escolar. La escuela privada, ¿contribuye de manera significativa a una mayor segregación escolar de los sistemas educativos? Y aquí interesa tanto la magnitud de la segregación de la educación privada como su aportación a la segregación global.

\subsection{La segregación escolar}

Entendemos por segregación escolar la desigual distribución de los estudiantes en las escuelas atendiendo a sus características personales o sociales (Jenkins, Micklewright y Schnepf, 2008; Murillo, 2016). De esta forma, es posible distinguir la segregación por nivel socioeconómico de las familias de los estudiantes, la segregación por el origen nativo o inmigrante de los estudiantes, la segregación por pertenencia a grupo cultural mayoritario o minoritario, pero también la segregación por rendimiento académico. En el presente trabajo, abordamos la segregación por nivel socioeconómico por su impacto en la equidad de los sistemas educativos (Benito, Alegre y González-Balletbò, 2014; Elacqua et al., 2016; Gorard, 2000). 
Los estudios de segregación escolar se preocuparon inicialmente de la segregación de carácter étnico-racial. Surgieron hacia finales de la década de los 50 tras el famoso caso Brown, contra el Consejo de Educación de Topeka de 1954, por el que la Corte Suprema de los Estados Unidos declaró ilegal la segregación de carácter étnico-racial en escuelas públicas norteamericanas (Coleman, Kelly y Moore, 1975; Reardon y Owens, 2014). Habría que esperar hasta los años 80 para que surjan con fuerza los trabajos que estiman la magnitud de la segregación escolar por nivel socioeconómico. Uno de los primeros trabajos fue el realizado por Wilson (1987), en donde se reflejan las desventajas de la concentración de familias de clases sociales más desfavorecidas en algunas escuelas. Desde entonces, un gran número de trabajos han cuantificado su magnitud (Gorard y Smith, 2004; Gorard y Taylor, 2002; Murillo, 2016; Orfiel y Lee, 2005; Stephan, 2013), analizado sus efectos (Rivkin, 2000) o estudiado su evolución en el tiempo (Gorard, 2009).

Hoy por hoy, la segregación escolar por nivel socioeconómico es reconocida como la principal causa de la fragmentación del sistema educativo y como una de las variables que más impacta en el rendimiento de los estudiantes (OCDE, 2013; UNESCO, 2005). De acuerdo con OCDE (2013), la segregación escolar es resultado de la combinación de factores estructurales y culturales de la sociedad que impactan clara y directamente sobre la escuela. Entre ellos, destacan la creciente segregación urbana (o residencial), el desarrollo de políticas educativas que favorecen la aplicación de la lógica de mercado a la educación, la tendencia a maximizar la autonomía de los centros y la búsqueda de distinción social.

El incremento de la oferta privada en el sector educativo es uno de los factores que aumentaron el interés por estudiar el origen, la magnitud y la evolución de la segregación escolar por nivel socioeconómico entre las escuelas públicas y privadas en diferentes países (Gorard y Smith, 2004; Orfiel y Lee, 2005; Stephan, 2013).

La investigación internacional apunta a que el nivel de segregación escolar entre las escuelas públicas y privadas es cada vez mayor en términos de desigualdad socioeconómica de sus estudiantes. Según Jenkins, Micklewright y Schnepf (2008), la magnitud promedio de la segregación escolar por nivel socioeconómico en las escuelas privadas es de 0,11 puntos y de 0,08 en las públicas, con datos PISA-2000 de 11 países de la OCDE. En ese estudio, sin embargo, no parece encontrarse relación entre el porcentaje de escuelas privadas en un país y su grado de segregación.

El nivel socioeconómico es un factor que tiene mayor incidencia en la segregación escolar que otros como la migración o la pertenencia a la etnia gitana (Síndic de Greuges, 2016). Sin embargo, en España son muy pocos los estudios que analizan la segregación escolar en las escuelas públicas y privadas bajo esta perspectiva. Entre ellos destacan los trabajos elaborados por Mancebón-Torrubia y Pérez-Ximénez de Embún (2008, 2010, 2015). Mancebón-Torrubia y Pérez-Ximénez de Embún (2008) subrayan una clara relación positiva entre el nivel socioeconómico y la probabilidad de asistir a un centro concertado. En 
concreto, un incremento del $1 \%$ en este indicador implica un aumento de un $5,9 \%$ en la probabilidad de asistir a centros concertados. Estos resultados coinciden con los encontrados por la profesora San Segundo (1991), quien demuestra que en las comunidades autónomas donde existe un mayor nivel de renta per cápita y mayor número de centros concertados existe mayor probabilidad de que un estudiante con recursos asista a un centro concertado.

En un estudio posterior, Mancebón-Torrubia y Pérez-Ximénez de Embún (2010) buscaron conocer cómo es la distribución socioeconómica del alumnado en los centros públicos y concertados de educación secundaria para conocer si existe segregación escolar por nivel socioeconómico entre estas escuelas. Los autores utilizan los datos de 19.604 estudiantes que participaron en PISA2006. Los resultados encontrados señalan que el nivel más elevado de segregación se alcanza en las escuelas concertadas de Andalucía, Cantabria, el País Vasco y Navarra (índice de segregación superior a 0,45). Las mayores diferencias socioeconómicas entre las propias escuelas públicas se dan en Galicia y el País Vasco (índices superiores a 0,30) (valores medidos a través del índice de disimilitud). Valores que contrastan con los resultados de Jenkins, Micklewright y Schnepf (2008), quienes, utilizando los datos PISA-2000, calculan que el nivel de segregación por nivel socioeconómico en las escuelas privadas españolas es de 0,173, y en las públicas de 0,096, valores medidos a través del índice de raíz cuadrada.

Respecto al análisis de la segregación escolar por nivel socioeconómico dentro de la circunscripción de regiones específicas, destacan los informes del Síndic de Greuges $(2008,2016)$ que trabajan el tema en la región de Cataluña. Estos informes resaltan la especial incidencia de estos factores relacionados con el origen social del alumnado en la segregación escolar. Dada su importancia, recomiendan desarrollar instrumentos para conocer de forma más ajustada la composición social de cada centro y para incidir en la distribución equitativa del alumnado en función de categorías sociales (nivel instructivo, nivel económico, etcétera), sin olvidar incluir la población con mayor capital económico y cultural.

\section{Método}

La presente investigación pretende, en primer lugar, estimar la magnitud de la segregación escolar por nivel socioeconómico de los centros de Educación Secundaria Obligatoria de titularidad pública y de titularidad privada en España y en cada comunidad autónoma y, en segundo lugar, determinar la aportación de los centros públicos y privados a la segregación total.

Para conseguir dicho objetivo se realiza una explotación secundaria de la base de datos española en la evaluación PISA-2015 (OCDE, 2016), datos liberados en diciembre de 2016 y los últimos disponibles. Concretamente, supone disponer de informaciones de un total de 39,066 estudiantes de 15 años escolarizados en 1.177 escuelas. De ellos, 6.736 integran la muestra que aporta datos del conjunto de Estado. Los 32.330 restantes aportan datos de 
Tabla 2. Muestra y sus características

\begin{tabular}{lccc}
\hline & No estudiantes $^{\text {No }}$ & $\begin{array}{c}\mathbf{N}^{\circ} \text { centros } \\
\text { educativos }\end{array}$ & $\begin{array}{c}\text { \% estudiantes en } \\
\text { centros públicos }\end{array}$ \\
\hline España & 6.736 & 201 & 68,61 \\
Andalucía & 1.813 & 54 & 74,53 \\
Aragón & 1.798 & 53 & 64,39 \\
Principado de Asturias & 1.790 & 54 & 65,87 \\
Islas Baleares & 1.797 & 54 & 63,61 \\
Canarias & 1.842 & 54 & 78,24 \\
Cantabria & 1.924 & 56 & 68,18 \\
Castilla y León & 1.858 & 57 & 64,47 \\
Castilla-La Mancha & 1.889 & 55 & 80,78 \\
Cataluña & 1.769 & 52 & 61,37 \\
Extremadura & 1.809 & 53 & 74,59 \\
Galicia & 1.865 & 59 & 72,92 \\
La Rioja & 1.461 & 47 & 59,52 \\
Comunidad de Madrid & 1.808 & 51 & 59,48 \\
Región de Murcia & 1.796 & 53 & 76,68 \\
C. Foral de Navarra & 1.874 & 52 & 62,42 \\
País Vasco & 3.612 & 119 & 52,66 \\
Comunidad Valenciana & 1.625 & 53 & 67,15 \\
Total/promedio & 39.066 & 1.177 & 68,61 \\
\hline
\end{tabular}

${ }^{*}$ Nota: datos ponderados.

Fuente: elaboración propia a partir de datos de PISA 2015.

cada una de las 17 comunidades, con una media de 1.800 estudiantes por comunidad, excepto el País Vasco que duplica su muestra (tabla 2).

Para la selección de esta muestra, PISA siguió un muestreo estratificado por conglomerados en dos etapas. Las variables de estratificación utilizadas para el caso español fueron la titularidad del centro, la comunidad autónoma, el tamaño de la escuela y la modalidad de enseñanza en el País Vasco, como variables explícitas, y los códigos postales y en Cataluña el tamaño de la localidad, como variables implícitas.

Las variables utilizadas son:

- Nivel socioeconómico y cultural de la familia del estudiante (NSEC), constructo estimado por PISA a partir de la información de las siguientes variables: índice socioeconómico internacional de situación laboral (ISEI), nivel educativo más alto de los padres del estudiante transformados en años de escolaridad, índice PISA de la riqueza de la familia, índice PISA de recursos educativos del hogar y, por último, el índice PISA de posesiones relacionadas con la cultura en el domicilio familiar. Variable tipificada para la muestra española.

- Titularidad del centro educativo en el que está matriculado el estudiante: público o privado. A los fines del análisis, se consideran privados tanto los 
centros financiados con fondos públicos como los no concertados, pues la cifra de centros privados no concertados en Educación Secundaria Obligatoria es tan pequeña (7 de los 1.177 centros de la muestra) que no merece hacer la diferenciación.

Ahora bien, para alcanzar el objetivo se requiere conocer la descomposición de la segregación escolar en la aportación de los subsistemas escolar público y privado. El índice más adecuado para ello es el llamado índice de raíz cuadrada o índice de Hutchens $(\mathrm{H})$, en honor al investigador estadounidense que lo propuso (Hutchens, 2001, 2004). Este índice destaca entre todos los propuestos por tener la propiedad de descomposición aditiva, lo que permite determinar la aportación de varios subconjuntos a la segregación total. Con ello, es el único que permite estimar la contribución de los subsistemas público y privado a la segregación global, que es el objetivo de este trabajo (Murillo, 2016).

El índice $\mathrm{H}$ se interpreta como la suma del alejamiento de cada escuela de la igualdad distributiva: la distancia entre la media geométrica de las participaciones de estudiantes de distinto nivel socioeconómico en ausencia de segregación y la media geométrica de las participaciones reales (Jenkins, Micklewright y Schnepf, 2008).

Se estima mediante la siguiente fórmula:

$$
H=\sum_{i=1}^{k}\left(\left(\frac{x_{1 i}}{X_{1}}\right)-\sqrt{\frac{x_{2 i}}{X_{2}} \frac{x_{1 i}}{X_{1}}}\right)
$$

Donde, para cada comunidad autónoma, $\mathrm{x}_{1 \mathrm{i}} \mathrm{y}_{2 \mathrm{i}}$ representan el número de alumnos del grupo minoritario y mayoritario, respectivamente, en la escuela i, $\mathrm{y}_{1}$ y $\mathrm{X}_{2}$ son el número total de estudiantes minoritarios y mayoritarios en todas las escuelas del país.

Por su propiedad de descomposición aditiva es posible dividir el índice en dos partes: la magnitud de la segregación para cada subsistema (intersectorial) y la segregación generada entre ambos conjuntamente (intrasectorial). El componente «H intrasectorial» es una suma ponderada de la segregación dentro de cada sector g; y el componente «H intersectorial» puede expresarse como fracción de $\mathrm{H}$ para evaluar qué proporción de la segregación total se debe a la distribución desigual por nivel socioeconómico entre un subsistema y otro, en este caso entre escuelas públicas y privadas.

$$
H=H_{\text {intra }}+H_{\text {inter }}
$$

Donde

$$
H_{\text {intra }}=\sum_{g=1}^{G} W_{g} H_{g}
$$


Con

$$
W_{g}=\sqrt{\left(\frac{P_{g}}{P}\right)\left(\frac{R_{g}}{R}\right)}
$$

Donde $\mathrm{g}=1 \ldots$, G subgrupos, $\mathrm{y} \mathrm{w}_{\mathrm{g}}$ es el peso del subgrupo $\mathrm{g}, \mathrm{P}_{\mathrm{g}} \mathrm{y} \mathrm{R}_{\mathrm{g}}$ el número de estudiantes en el subgrupo $\mathrm{g}$ con respecto al grupo minoritario $\mathrm{y}$ mayoritario P y R.

Dado que buscamos tener una imagen completa de la aportación de la educación pública y privada a la segregación global, se ha optado por trabajar con cuatro consideraciones de grupo minoritario. En primer lugar, se tuvo en cuenta el $10 \%$ de los estudiantes cuyas familias poseen el menor nivel socioeconómico y cultural (P10); en segundo término, se trabajó con el 25\% de los estudiantes con familias con menor nivel socioeconómico y cultural (Q1); en tercer lugar, con el 25\% de los estudiantes con familias de mayor NSEC (Q4). Por último, se decidió considerar también el 10\% de los estudiantes que pertenecen a familias con mayor NSEC (P90). La razón es que la segregación se produce tanto entre los estudiantes más vulnerables como entre los menos, pero su incidencia es diferente entre centros públicos y privados.

\section{Resultados}

Antes de presentar los hallazgos que dan respuesta al objetivo de esta investigación - estimar la magnitud de la segregación de los centros públicos y privados y su aportación diferencial a la segregación escolar-, vamos a presentar dos resultados previos que ayudarán a comprender lo encontrado. Por una parte, se ofrecerá una panorámica de la segregación escolar en España y sus comunidades; y, por otra, se presentará una imagen de la distribución de los estudiantes según su nivel socioeconómico entre centros públicos y privados.

Con ello se ofrecerá un minucioso análisis de la descomposición de la segregación escolar por nivel socioeconómico en centros públicos y privados para diferentes grupos minoritarios. En primer lugar, se ofrecen los datos para el 10\% de los estudiantes cuyas familias tienen menor nivel socioeconómico y cultural (P10), después para el 25\% de menor NSEC (Q1), a continuación, para el 25\% de mayor NSEC (Q4) y para finalizar para el 10\% con mayor NSEC (P90). En todos los casos, presentaremos inicialmente los datos sin ponderar, es decir, la segregación bruta de las escuelas públicas y privadas, y luego consideraremos su distribución en cada comunidad autónoma. Finalizamos los resultados con una visión general de la segregación en centros públicos y privados para todos los colectivos.

\subsection{Panorámica de la segregación escolar por comunidades y colectivos}

Antes de considerar la aportación de los subsistemas público y privado a la segregación escolar por nivel socioeconómico en Educación Secundaria Obli- 
Tabla 3. Segregación escolar por nivel socioeconómico en educación secundaria en España para diferentes colectivos. Índice de raíz cuadrada para el P10, Q1, Q4 y P90 en NSEC como grupos minoritarios, por comunidad autónoma

\begin{tabular}{lcccc}
\hline & $\begin{array}{c}10 \% \text { de los } \\
\text { estudiantes } \\
\text { con menor } \\
\text { NSEC }\end{array}$ & $\begin{array}{c}25 \% \text { de los } \\
\text { estudiantes } \\
\text { con menor } \\
\text { NSEC }\end{array}$ & $\begin{array}{c}25 \% \text { de los } \\
\text { estudiantes } \\
\text { con mayor } \\
\text { NSEC }\end{array}$ & $\begin{array}{c}10 \% \text { de los } \\
\text { estudiantes } \\
\text { con mayor } \\
\text { NSEC }\end{array}$ \\
\hline España & 0,2234 & 0,1734 & 0,1954 & 0,2458 \\
Andalucía & 0,1452 & 0,1278 & 0,1351 & 0,2430 \\
Aragón & 0,1488 & 0,1032 & 0,1058 & 0,1584 \\
Principado de Asturias & 0,1861 & 0,1312 & 0,1789 & 0,2480 \\
Islas Baleares & 0,1260 & 0,0838 & 0,0872 & 0,1343 \\
Canarias & 0,1603 & 0,1160 & 0,1901 & 0,2993 \\
Cantabria & 0,1310 & 0,0855 & 0,1163 & 0,1956 \\
Castilla y León & 0,1090 & 0,0798 & 0,1359 & 0,2145 \\
Castilla-La Mancha & 0,1588 & 0,1082 & 0,1704 & 0,2097 \\
Cataluña & 0,1973 & 0,1503 & 0,2004 & 0,2063 \\
Extremadura & 0,1211 & 0,0856 & 0,1114 & 0,2092 \\
Galicia & 0,0977 & 0,1009 & 0,1244 & 0,1677 \\
La Rioja & 0,1674 & 0,1196 & 0,0878 & 0,1810 \\
Comunidad de Madrid & 0,2771 & 0,2047 & 0,2281 & 0,2325 \\
Región de Murcia & 0,1558 & 0,0999 & 0,1138 & 0,1591 \\
C. Foral de Navarra & 0,2127 & 0,1062 & 0,1217 & 0,1743 \\
País Vasco & 0,2096 & 0,1263 & 0,1393 & 0,2086 \\
Comunidad Valenciana & 0,1605 & 0,1044 & 0,1140 & 0,1767 \\
\hline Funte: & & &
\end{tabular}

Fuente: elaboración propia a partir de los datos de PISA-2012.

gatoria en España, es interesante analizar la magnitud de la segregación en cada comunidad autónoma para cada colectivo minoritario considerado. Los resultados, utilizando el índice de raíz cuadrada de Hutchens, se muestran en la tabla 3 .

Dos ideas principales surgen del análisis de los resultados. En primer lugar, que la magnitud de la segregación depende directamente del grupo minoritario considerado. De esta forma, para el conjunto de España y para la mayoría de las comunidades (pero no para todas), la puntuación más alta en el nivel de segregación se da para el 10\% de estudiantes cuyos padres tienen mayor nivel socioeconómico y cultural (P90); seguido del 10\% de los que menos tienen (P10), del 25\% de los que menos (Q1) y del 25\% de los que más (Q4). Como señalamos, esta tendencia general no se da en todas las comunidades, pero el índice de segregación para el P90, que alcanza el 0,22 para el conjunto de España, demuestra que el problema de la segregación escolar es real y amerita atención.

La segunda idea que surge del análisis es que las diferencias de una comunidad a otra son muy importantes. Tanto que la Comunidad de Madrid, que es la región globalmente más segregadora, multiplica por 2,4 sus índices respecto a las Islas Baleares, la región con menor segregación. Concretamente, el índice 
Gráfico 1. Segregación escolar por nivel socioeconómico en educación secundaria en España para diferentes colectivos. Índice de raíz cuadrada para el P10, Q1, Q4 y P90 en NSEC como grupos minoritarios, por comunidad autónoma

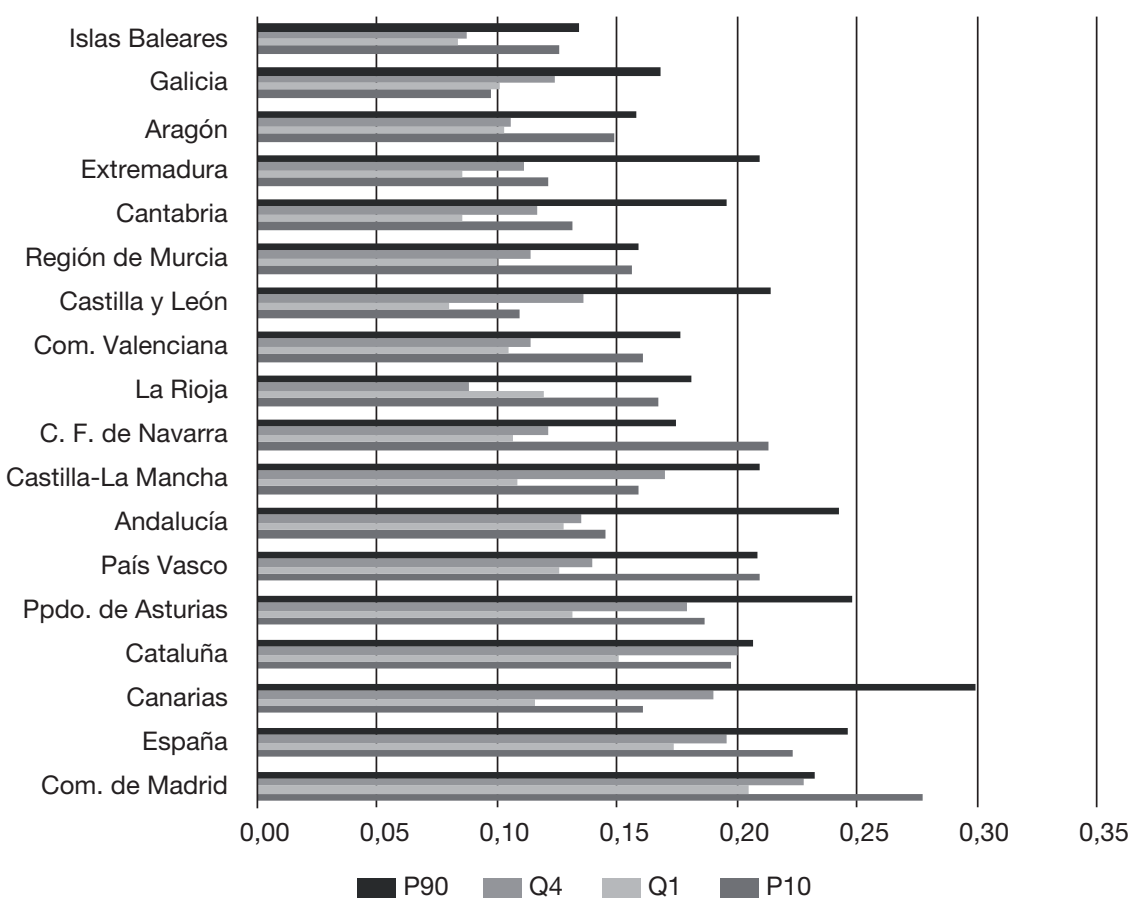

Nota: comunidades ordenadas por el promedio de los índices de segregación.

Fuente: elaboración propia a partir de los datos de PISA-2015.

de segregación alcanza un valor de 0,24 para la Comunidad de Madrid y de 0,10 para las Islas Baleares. La heterogeneidad entre los índices de las diferentes comunidades demuestra que el problema de la segregación escolar en España es, a su vez, complejo, por lo que requiere el conocimiento diferenciado de cada situación.

Sin embargo, como se aprecia en el gráfico 1, la segregación escolar de cada comunidad autónoma tiene características diferentes. Solo a modo de ejemplo, mientras que en la Comunidad de Madrid o la Comunidad Foral de Navarra la segregación es especialmente alta para los estudiantes de familias con mayor nivel socioeconómico (P90), en Canarias, Asturias o Andalucía, se observa que la segregación más alta se da entre el 10\% de estudiantes cuyas familias tienen menor nivel socioeconómico (P10).

Estos resultados, sin duda, afectan a la descomposición de la segregación entre los sistemas público y privado, haciendo más interesante y complejo el tema objeto de estudio en esta investigación. 


\subsection{Distribución de los estudiantes de cada colectivo en centros públicos $y$ privados}

Para entender la descomposición de la segregación entre los subsistemas público y privado se necesita conocer la distribución de los estudiantes de Educación Secundaria Obligatoria entre centros públicos y privados para cada grupo analizado. La tabla 4 presenta esta información.

Como puede observarse, y ya constituye un hallazgo interesante, la probabilidad de estudiar en un centro público aumenta cuanto menor es el nivel socioeconómico y cultural de la familia del estudiante. Para toda España, el $88,4 \%$ de los estudiantes del $10 \%$ con familias de menor nivel socioeconómico y cultural estudian en centros públicos, mientras que esta cifra es del 36,3\% para el 10\% con mayor nivel socioeconómico. La diferencia de más del 50\% entre uno y otro grupo poblacional advierte sobre cuán determinante es el factor socioeconómico en la elección de la titularidad del centro.

Aún más interesante es ver que la distribución de la educación públicaprivada es diferente en cada comunidad y para cada colectivo. Puede observarse, por ejemplo, que La Rioja es donde hay mayor porcentaje de estudiantes del P10 escolarizados en centros privados $(31,2 \%)$ y Castilla-La Mancha donde menos (2,8\%). Mientras que para el P90 la autonomía con mayor

Tabla 4. Porcentaje de estudiantes de Educación Secundaria Obligatoria escolarizados en centros públicos del $10 \%$ y $20 \%$ de los estudiantes con familias de menor nivel socioeconómico y cultural (P10 y Q1) y del 10\% y $25 \%$ de mayor nivel socioeconómico y cultural (P90 y Q4), por comunidad autónoma

\begin{tabular}{lcccc}
\hline & $\begin{array}{c}\text { \% en centros } \\
\text { públicos del P10 }\end{array}$ & $\begin{array}{c}\text { \% en centros } \\
\text { públicos del Q1 }\end{array}$ & $\begin{array}{c}\text { \% en centros } \\
\text { públicos del Q4 }\end{array}$ & $\begin{array}{c}\% \text { en centros } \\
\text { públicos del P90 }\end{array}$ \\
\hline España & 88,36 & 86,25 & 43,06 & 36,30 \\
Andalucía & 87,68 & 84,86 & 56,36 & 50,16 \\
Aragón & 77,47 & 77,54 & 49,36 & 45,27 \\
Principado de Asturias & 81,90 & 79,89 & 44,07 & 39,45 \\
Islas Baleares & 73,72 & 72,03 & 53,41 & 49,86 \\
Canarias & 94,94 & 94,12 & 49,51 & 35,61 \\
Cantabria & 80,72 & 77,23 & 54,70 & 49,61 \\
Castilla y León & 75,57 & 76,63 & 48,25 & 44,67 \\
Castilla-La Mancha & 97,16 & 95,57 & 55,51 & 46,86 \\
Cataluña & 86,53 & 80,59 & 33,90 & 28,67 \\
Extremadura & 89,94 & 87,99 & 55,96 & 47,32 \\
Galicia & 86,52 & 83,24 & 56,60 & 49,33 \\
La Rioja & 72,53 & 75,61 & 48,04 & 45,58 \\
Comunidad de Madrid & 81,16 & 78,47 & 41,23 & 35,49 \\
Región de Murcia & 94,89 & 89,47 & 60,73 & 56,90 \\
C. Foral de Navarra & 88,81 & 82,15 & 39,06 & 35,33 \\
País Vasco & 68,75 & 64,17 & 37,41 & 29,93 \\
Comunidad Valenciana & 77,32 & 77,22 & 52,50 & 44,32 \\
\hline
\end{tabular}

Fuente: elaboración propia a partir de los datos de PISA-2015. 
peso de la privada es Cataluña, con un 72,79\% de estudiantes, y la de menor peso es la Región de Murcia, con un 43,1\% de los estudiantes en centros privados.

\subsection{Segregación en escuelas públicas y privadas}

El índice de raíz cuadrada (o de Hutchens) posibilita descomponer la segregación escolar total en los subsistemas que la componen, en este caso, el subsistema de titularidad pública y de titularidad privada. Así, la segregación total es la aportación de la segregación de cada subsistema a la misma más la segregación intersubsistemas. Y esta aportación es la segregación «bruta» de cada subsistema ponderada por su tamaño relativo respecto a la población total. En este apartado, estimaremos primero la segregación bruta — sin ponderar - para cada uno de los cuatro colectivos de estudio y luego la aportación de la segregación de cada subsistema al total. De esta forma daremos respuesta a los dos objetivos planteados.

Todas las estimaciones para el 10\% de estudiantes con familias de menor nivel socioeconómico se presentan en la tabla 5.

Tabla 5. Segregación socioeconómica en centros educativos públicos y privados de educación secundaria en España. Descomposición del índice de raíz cuadrada para el 10\% de los estudiantes cuyas familias tienen menor nivel socioeconómico y cultural (P10) como grupo minoritario, por comunidad autónoma

\begin{tabular}{|c|c|c|c|c|c|c|c|c|}
\hline & \multirow[b]{3}{*}{$\mathrm{H}$ total } & \multicolumn{6}{|c|}{$\mathrm{H}$ intrasubsistemas } & \multirow{3}{*}{$\begin{array}{c}\text { H inter- } \\
\text { subsistemas }\end{array}$} \\
\hline & & \multicolumn{3}{|c|}{ Centros ed. públicos } & \multicolumn{3}{|c|}{ Centros ed. privados } & \\
\hline & & $\mathrm{H}$ bruto & Pond. & Aport. & $\mathrm{H}$ bruto & Pond. & Aport. & \\
\hline España & 0,2234 & 0,1375 & 0,7651 & 0,1052 & 0,4142 & 0,1975 & 0,0818 & 0,0364 \\
\hline Andalucía & 0,1452 & 0,0803 & 0,7811 & 0,0627 & 0,3678 & 0,1778 & 0,0654 & 0,0171 \\
\hline Aragón & 0,1488 & 0,0936 & 0,6668 & 0,0624 & 0,2658 & 0,2758 & 0,0733 & 0,0131 \\
\hline Ppdo. de Asturias & 0,1861 & 0,0865 & 0,6633 & 0,0574 & 0,4261 & 0,2328 & 0,0992 & 0,0295 \\
\hline Islas Baleares & 0,1260 & 0,1049 & 0,6787 & 0,0712 & 0,1513 & 0,3140 & 0,0475 & 0,0073 \\
\hline Canarias & 0,1603 & 0,1030 & 0,8375 & 0,0863 & 0,3247 & 0,1075 & 0,0349 & 0,0392 \\
\hline Cantabria & 0,1310 & 0,0668 & 0,7111 & 0,0475 & 0,2735 & 0,2450 & 0,0670 & 0,0165 \\
\hline Castilla y León & 0,1090 & 0,0677 & 0,6554 & 0,0444 & 0,1559 & 0,2842 & 0,0443 & 0,0203 \\
\hline Castilla-La Mancha & 0,1588 & 0,0964 & 0,8758 & 0,0844 & 0,3558 & 0,0773 & 0,0275 & 0,0468 \\
\hline Cataluña & 0,1973 & 0,0532 & 0,7119 & 0,0379 & 0,4554 & 0,2363 & 0,1076 & 0,0519 \\
\hline Extremadura & 0,1211 & 0,0788 & 0,8096 & 0,0638 & 0,1943 & 0,1652 & 0,0321 & 0,0252 \\
\hline Galicia & 0,0977 & 0,0561 & 0,7752 & 0,0435 & 0,1785 & 0,1938 & 0,0346 & 0,0196 \\
\hline La Rioja & 0,1674 & 0,0970 & 0,5947 & 0,0577 & 0,2962 & 0,3109 & 0,0921 & 0,0176 \\
\hline Com. de Madrid & 0,2771 & 0,1880 & 0,6805 & 0,1279 & 0,4012 & 0,2844 & 0,1141 & 0,0351 \\
\hline Región de Murcia & 0,1558 & 0,0899 & 0,8416 & 0,0757 & 0,3120 & 0,1138 & 0,0355 & 0,0446 \\
\hline C. F. de Navarra & 0,2127 & 0,0953 & 0,7185 & 0,0685 & 0,3027 & 0,2108 & 0,0638 & 0,0280 \\
\hline País Vasco & 0,2096 & 0,1418 & 0,4662 & 0,0661 & 0,2025 & 0,3092 & 0,0626 & 0,0275 \\
\hline Com. Valenciana & 0,1605 & 0,0928 & 0,7145 & 0,0663 & 0,2248 & 0,2776 & 0,0624 & 0,0282 \\
\hline
\end{tabular}

Fuente: elaboración propia a partir de los datos de PISA-2015. 
Los datos indican que, tanto para el $10 \%$ de los estudiantes cuyas familias tienen menor nivel socioeconómico y cultural como para el conjunto de España, las escuelas privadas segregan considerablemente más que las públicas. Concretamente, el índice de raíz cuadrada «bruto» para P10 para los centros educativos públicos de Educación Secundaria Obligatoria es de 0,14 , mientras que los centros privados casi triplican esta cifra, alcanzando el 0,41 .

Este comportamiento, sin embargo, es considerablemente diferente de una comunidad a otra. En primer lugar, encontramos que la segregación escolar para el $10 \%$ de los estudiantes con familias de menor nivel socioeconómico del subsistema de educación pública es globalmente y en comparación baja. Las variaciones oscilan entre 0,05 de Cataluña y 0,19 de la Comunidad de Madrid, con la mayoría de las comunidades por debajo de 0,10 (gráfico 2). Frente a la heterogeneidad de situaciones, hay una constante por la cual los centros públicos sostienen niveles bajos de segregación de los estudiantes más vulnerables. En una situación donde predomina la variabilidad, destaca esta característica que es sostenida por todas las comunidades autónomas.

La segregación en los centros privados, por el contrario, es mucho más alta que la segregación de los centros privados para el 10\% de los estudiantes de menor nivel socioeconómico. Además, muestra un gran rango de variación, pues es muy dispar entre unas comunidades y otras. En concreto, es:

Gráfico 2. Segregación socioeconómica, en valores absolutos, de los centros educativos públicos y privados de Educación Secundaria Obligatoria en España. Índice de raíz cuadrada para el $10 \%$ de los estudiantes con familias con menor nivel socioeconómico y cultural (P10) como grupo minoritario, por comunidades autónomas

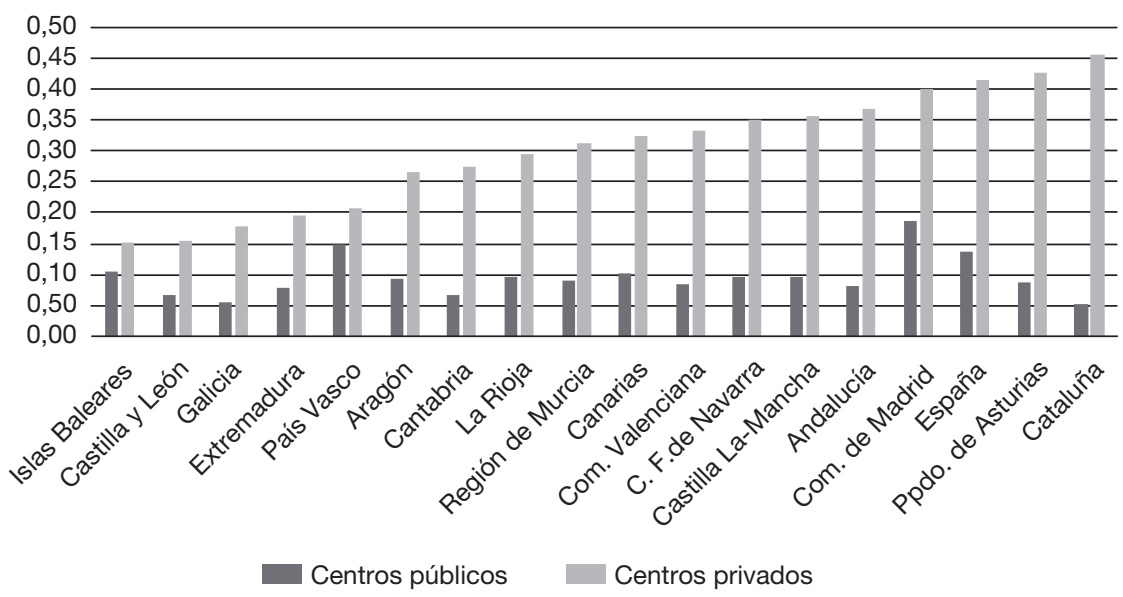

Nota: comunidades ordenadas por su índice de segregación en escuelas privadas.

Fuente: elaboración propia a partir de los datos de PISA-2015. 
Tabla 6. Segregación socioeconómica en centros educativos públicos y privados de Educación Secundaria Obligatoria en España. Descomposición del índice de raíz cuadrada para el $25 \%$ de los estudiantes con familias con menor nivel socioeconómico y cultural (Q1) como grupo minoritario, por comunidad autónoma

\begin{tabular}{|c|c|c|c|c|c|c|c|c|}
\hline & \multirow[b]{3}{*}{$\mathrm{H}$ total } & \multicolumn{6}{|c|}{$\mathrm{H}$ intrasubsistemas } & \multirow{3}{*}{$\begin{array}{c}\mathrm{H} \text { inter- } \\
\text { subsistemas }\end{array}$} \\
\hline & & \multicolumn{3}{|c|}{ Centros ed. públicos } & \multicolumn{3}{|c|}{ Centros ed. privados } & \\
\hline & & $\mathrm{H}$ bruto & Pond. & Aport. & $\mathrm{H}$ bruto & Pond. & Aport. & \\
\hline España & 0,1734 & 0,0907 & 0,7351 & 0,0667 & 0,3033 & 0,2262 & 0,0686 & 0,0380 \\
\hline Andalucía & 0,1278 & 0,0621 & 0,7570 & 0,0470 & 0,3250 & 0,2037 & 0,0662 & 0,0146 \\
\hline Aragón & 0,1032 & 0,0372 & 0,6534 & 0,0243 & 0,2121 & 0,2867 & 0,0608 & 0,0181 \\
\hline Ppdo. de Asturias & 0,1312 & 0,0694 & 0,6512 & 0,0452 & 0,2309 & 0,2590 & 0,0598 & 0,0262 \\
\hline Islas Baleares & 0,0838 & 0,0579 & 0,6618 & 0,0383 & 0,1160 & 0,3311 & 0,0384 & 0,0071 \\
\hline Canarias & 0,1160 & 0,0544 & 0,8149 & 0,0443 & 0,1945 & 0,1244 & 0,0242 & 0,0475 \\
\hline Cantabria & 0,0855 & 0,0333 & 0,6900 & 0,0230 & 0,1823 & 0,2742 & 0,0500 & 0,0126 \\
\hline Castilla y León & 0,0798 & 0,0409 & 0,6456 & 0,0264 & 0,1086 & 0,2891 & 0,0314 & 0,0220 \\
\hline Castilla-La Mancha & 0,1082 & 0,0586 & 0,8513 & 0,0499 & 0,1266 & 0,1035 & 0,0131 & 0,0452 \\
\hline Cataluña & 0,1503 & 0,0503 & 0,6655 & 0,0335 & 0,2634 & 0,2957 & 0,0779 & 0,0388 \\
\hline Extremadura & 0,0856 & 0,0517 & 0,7854 & 0,0406 & 0,1051 & 0,1894 & 0,0199 & 0,0251 \\
\hline Galicia & 0,1009 & 0,0530 & 0,7495 & 0,0397 & 0,1885 & 0,2234 & 0,0421 & 0,0192 \\
\hline La Rioja & 0,1196 & 0,0527 & 0,5886 & 0,0310 & 0,1834 & 0,3076 & 0,0564 & 0,0322 \\
\hline Com. de Madrid & 0,2047 & 0,1536 & 0,6457 & 0,0992 & 0,2166 & 0,3176 & 0,0688 & 0,0366 \\
\hline Región de Murcia & 0,0999 & 0,0621 & 0,8049 & 0,0500 & 0,1479 & 0,1704 & 0,0252 & 0,0247 \\
\hline C. F. de Navarra & 0,1062 & 0,0659 & 0,6664 & 0,0439 & 0,2238 & 0,2768 & 0,0469 & 0,0272 \\
\hline País Vasco & 0,1263 & 0,0967 & 0,4385 & 0,0424 & 0,2205 & 0,3361 & 0,0454 & 0,0265 \\
\hline Com. Valenciana & 0,1044 & 0,0600 & 0,7018 & 0,0421 & 0,2144 & 0,2872 & 0,0440 & 0,0273 \\
\hline
\end{tabular}

Fuente: elaboración propia a partir de los datos de PISA-2015.

- Muy alto (más de 0,4) en Cataluña $(0,46)$, el Principado de Asturias $(0,43)$ y la Comunidad de Madrid $(0,40)$, así como para el conjunto de España $(0,41)$.

- Alto (entre 0,3 y 0,4) en Andalucía (0,37), Castilla-La Mancha (0,36), la Comunidad Foral de Navarra $(0,35)$, la Comunidad Valenciana $(0,33)$, Canarias $(0,32)$ y la Región de Murcia $(0,31)$.

- Alto-moderado (entre 0,2 y 0,3$)$ en La Rioja $(0,30)$, Cantabria $(0,27)$, Aragón $(0,27)$ y el País Vasco $(0,21)$.

- Moderado (menos de 0,2) en Extremadura (0,19), Galicia $(0,18)$, Castilla y León $(0,16)$ y las Islas Baleares $(0,15)$.

De esta forma, el análisis conjunto de la segregación en centros públicos y privados muestra interesantes matices y grandes diferencias en cada comunidad. Si comparamos los índices de segregación de los centros privados, encontramos que son 8,5 veces más altos que los índices de segregación de los centros públicos en Cataluña y cinco veces más altos en Asturias, mientras que en las Islas Baleares o en el País Vasco la diferencia es pequeña. 
Dado que, como hemos visto, en cada comunidad autónoma la distribución de estudiantes matriculados en centros públicos y privados es muy diferente, la descomposición de la segregación global en los dos subsistemas muestra otra cara de la realidad (tabla 5). En términos absolutos, la segregación de los centros privados para el P10 de los estudiantes es mucho mayor que la de los públicos, pero su aportación a la segregación global es menor debido a que hay pocos estudiantes matriculados en ellos. Para el total de España, la comentada magnitud de 0,2234 se descompone en 0,1052 que aporta el subsistema educativo público, 0,0818 que aporta el subsistema privado y 0,0364 que es la segregación intersubsistemas. Esta última, recordamos, se debe a la conexión entre ambos, es decir, a la posibilidad de movilidad entre los dos. Por comunidades, en la mayoría de los casos la segregación del subsistema público aporta más a la segregación global, sin embargo, en Cataluña, el Principado de Asturias, La Rioja, Cantabria, Aragón y Andalucía, la aportación del subsistema de centros privados a la segregación total es mayor que la de centros públicos.

En resumen, y respondiendo al objetivo de la investigación, para el 10\% de los estudiantes con menor nivel socioeconómico y cultural, el subsistema público contribuye a la segregación global más que el privado, pero esta afirmación esconde que también absorbe el mayor porcentaje de estudiantes vulnerables, lo que aumenta su peso relativo. En efecto, al considerar la segregación de cada subsistema de manera diferencial, se halla que el índice de los centros de titularidad privada es enormemente más alto que el de la pública para este grupo poblacional. Dentro de la heterogeneidad de situaciones que presenta cada comunidad autónoma, el problema es especialmente grave para la Comunidad de Madrid, cuyo índice de segregación es de 0,28 y la segregación de la educación pública, de 0,13 .

El análisis de la segregación público-privada para el 25\% de los estudiantes cuyas familias tienen un menor nivel socioeconómico y cultural (Q1) nos ofrece una panorámica análoga a la encontrada para el P10: la segregación «bruta» de los centros educativos privados es de nuevo mucho mayor que la de los centros públicos. Esta afirmación se aplica para el conjunto del Estado y también para todas y cada una de las comunidades (tabla 6).

Sin embargo, también aquí hay grandes diferencias entre comunidades (gráfico 3). En Andalucía, en un extremo, mientras que la segregación del subsistema público es de 0,06, la del subsistema privado alcanza el 0,32, es decir, 5,2 veces más. En la Comunidad de Madrid, en el otro extremo, la gran segregación que tienen los centros públicos hace que la diferencia entre ambas solo sea de 1,7 veces (de 0,15 para centros públicos y 0,21 para privados).

Las cifras cambian sustancialmente cuando se las pondera teniendo en cuenta el peso de la educación pública y privada en cada comunidad. Para el conjunto del Estado, así como para 12 de las 17 comunidades autónomas, la aportación del subsistema privado a la segregación total es mayor que la del público. Esta tendencia es eludida por Castilla-La Mancha, Comunidad de Madrid, Región de Murcia, Extremadura y Canarias, donde el subsistema público aporta más a la segregación que el privado; aunque también con gran- 
Gráfico 3. Segregación socioeconómica, en valores absolutos, de centros educativos públicos y privados de Educación Secundaria Obligatoria en España. Índice de raíz cuadrada para el $25 \%$ de los estudiantes con familias con menor nivel socioeconómico y cultural (Q1) como grupo minoritario, por comunidades

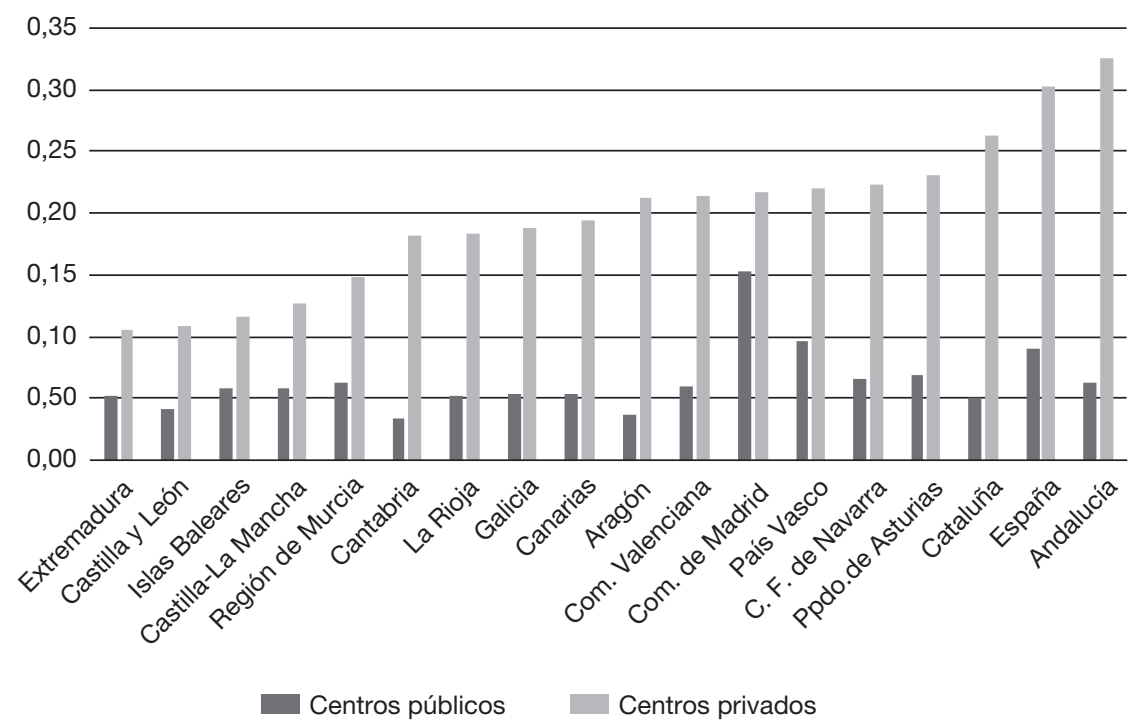

Nota: comunidades ordenadas por su índice de segregación en escuelas privadas.

Fuente: elaboración propia a partir de los datos de PISA-2015.

des diferencias de magnitud entre ellas. La aportación de los centros privados a la segregación es especialmente alta en Cataluña, con 0,08 en el índice de discriminación, y en la Comunidad de Madrid, con 0,07 puntos. El estudio diferenciado del 10\% (P10) y del 25\% (Q1) de los estudiantes con familias de menor nivel socioeconómico y cultural establece aquí una diferencia interesante, pues alternativamente la educación pública y la privada generan mayores índices de segregación en uno y otro grupo, lo que se debe fundamentalmente a la diferencia en el porcentaje de estudiantes que absorbe cada subsistema.

El análisis de la descomposición de segregación escolar por nivel socioeconómico y cultural para los subsistemas públicos y privados para el $25 \%$ de los estudiantes con familias de mayor NSEC es muy diferente al encontrado para el 25\% de menos NSEC. Para el total estatal y para cinco de las 17 comunidades, la segregación en los centros públicos es mayor que en los privados. Un análisis minucioso indica:

1. En el Principado de Asturias, Cataluña, Cantabria, Andalucía, Aragón, Canarias y la Comunidad Valenciana, la segregación de los centros privados es considerablemente más alta que en los públicos (de más de 0,06 puntos de diferencia). 
Gráfico 4. Segregación socioeconómica, en valores absolutos, de centros educativos públicos y privados de Educación Secundaria Obligatoria en España. Índice de raíz cuadrada para el $25 \%$ de los estudiantes con familias con mayor nivel socioeconómico y cultural (Q4) como grupo minoritario, por comunidades

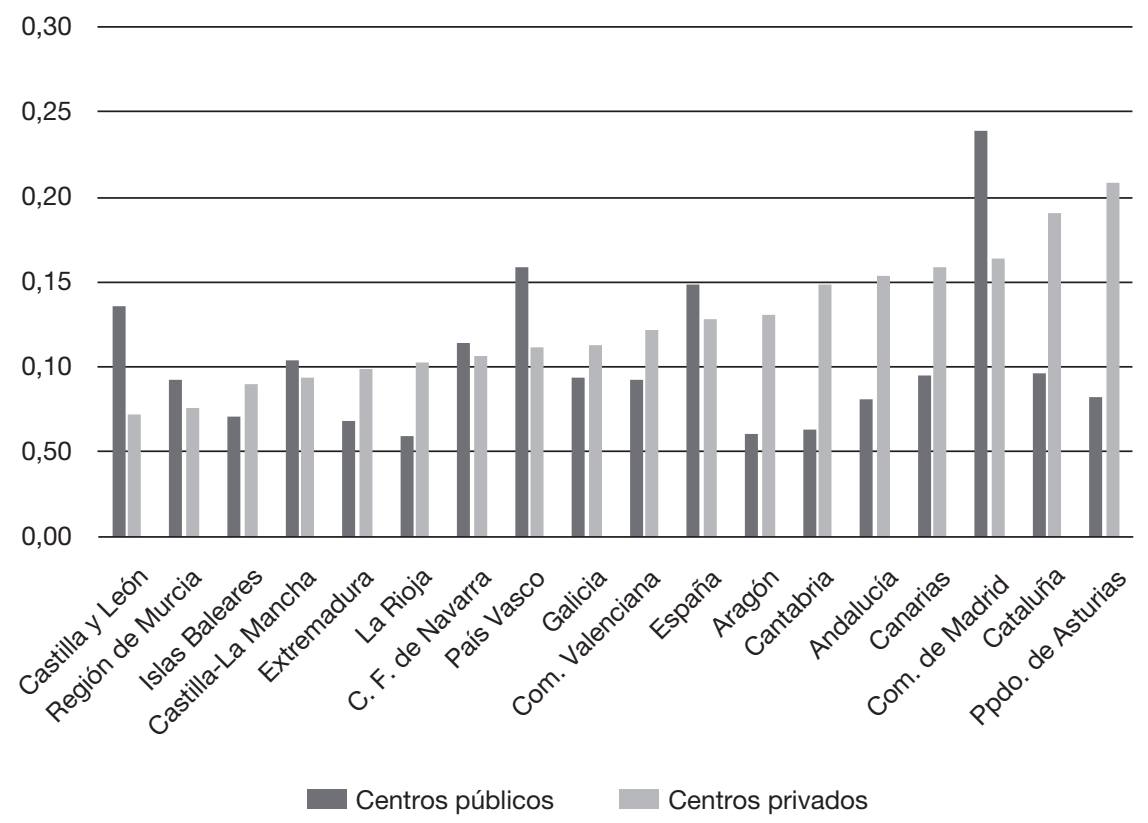

Nota: comunidades ordenadas por su índice de segregación en escuelas privadas.

Fuente: elaboración propia a partir de los datos de PISA-2015.

2. En La Rioja, Navarra y Extremadura es baja (entre 0,6 y 0,2).

3. En Galicia, las Islas Baleares, Castilla-La Mancha, el País Vasco y la Región de Murcia, las diferencias, en un sentido u otro, son muy pequeñas (de menos de 0,02 puntos).

4. En Castilla y León y la Comunidad de Madrid, las diferencias son grandes (superiores a 0,6) y más altas para los centros públicos.

El análisis de la descomposición de la segregación total en los subsistemas públicos y privados e intersistemas se muestra en la tabla 7. Como puede observarse, tanto en el total del Estado como en 12 de las 17 comunidades, la aportación de los centros públicos a la segregación global es mayor que la de los centros privados. Las excepciones son Cataluña, el Principado de Asturias, Aragón, Cantabria y La Rioja, donde la aportación de los centros privados es mayor.

Una última mirada nos aporta el estudio de la segregación para el 10\% de los estudiantes cuyas familias tienen mayor nivel socioeconómico y cultural (P90). En ese caso, para el conjunto de España, el subsistema de educación 
Tabla 7. Segregación socioeconómica en centros educativos públicos y privados de Educación Secundaria Obligatoria en España. Descomposición del índice de raíz cuadrada para el $25 \%$ de los estudiantes con familias con mayor nivel socioeconómico y cultural (Q4) como grupo minoritario, por comunidad autónoma

\begin{tabular}{|c|c|c|c|c|c|c|c|c|}
\hline & \multirow[b]{3}{*}{$\mathrm{H}$ total } & \multicolumn{6}{|c|}{$\mathrm{H}$ intrasubsistemas } & \multirow{3}{*}{$\begin{array}{c}\text { H inter- } \\
\text { subsiste-mas }\end{array}$} \\
\hline & & \multicolumn{3}{|c|}{ Centros ed. públicos } & \multicolumn{3}{|c|}{ Centros ed. privados } & \\
\hline & & $\mathrm{H}$ bruto & Pond. & Aport. & $\mathrm{H}$ bruto & Pond. & Aport. & \\
\hline España & 0,1954 & 0,1488 & 0,5761 & 0,0857 & 0,1286 & 0,3607 & 0,0464 & 0,0632 \\
\hline Andalucía & 0,1351 & 0,0814 & 0,6625 & 0,0539 & 0,1536 & 0,2852 & 0,0438 & 0,0375 \\
\hline Aragón & 0,1058 & 0,0604 & 0,5649 & 0,0341 & 0,1307 & 0,3794 & 0,0496 & 0,0221 \\
\hline Ppdo. de Asturias & 0,1789 & 0,0828 & 0,5422 & 0,0449 & 0,2082 & 0,3669 & 0,0764 & 0,0576 \\
\hline Islas Baleares & 0,0872 & 0,0707 & 0,5982 & 0,0423 & 0,0898 & 0,3921 & 0,0352 & 0,0097 \\
\hline Canarias & 0,1901 & 0,0948 & 0,6447 & 0,0611 & 0,1582 & 0,2433 & 0,0385 & 0,0904 \\
\hline Cantabria & 0,1163 & 0,0634 & 0,6086 & 0,0386 & 0,1484 & 0,3404 & 0,0505 & 0,0272 \\
\hline Castilla y León & 0,1359 & 0,1354 & 0,5430 & 0,0735 & 0,0720 & 0,3710 & 0,0267 & 0,0357 \\
\hline Castilla-La Mancha & 0,1704 & 0,1035 & 0,7036 & 0,0728 & 0,0943 & 0,2195 & 0,0207 & 0,0770 \\
\hline Cataluña & 0,2004 & 0,0957 & 0,4890 & 0,0468 & 0,1903 & 0,4414 & 0,0840 & 0,0696 \\
\hline Extremadura & 0,1114 & 0,0683 & 0,6724 & 0,0459 & 0,0987 & 0,2908 & 0,0287 & 0,0368 \\
\hline Galicia & 0,1244 & 0,0931 & 0,6478 & 0,0603 & 0,1129 & 0,2995 & 0,0338 & 0,0304 \\
\hline La Rioja & 0,0878 & 0,0588 & 0,5015 & 0,0295 & 0,1020 & 0,3981 & 0,0406 & 0,0176 \\
\hline Com. de Madrid & 0,2281 & 0,2389 & 0,5199 & 0,1242 & 0,1638 & 0,4499 & 0,0737 & 0,0302 \\
\hline Región de Murcia & 0,1138 & 0,0921 & 0,7056 & 0,0650 & 0,0763 & 0,2659 & 0,0203 & 0,0284 \\
\hline C. F. de Navarra & 0,1217 & 0,1138 & 0,5150 & 0,0586 & 0,1066 & 0,4183 & 0,0446 & 0,0422 \\
\hline País Vasco & 0,1393 & 0,1587 & 0,3579 & 0,0568 & 0,1119 & 0,3978 & 0,0445 & 0,0408 \\
\hline Com. Valenciana & 0,1140 & 0,0925 & 0,6149 & 0,0569 & 0,1221 & 0,3645 & 0,0445 & 0,0411 \\
\hline
\end{tabular}

Fuente: elaboración propia a partir de los datos de PISA-2015.

público segrega en mayor medida que el privado. En valores brutos, el índice $\mathrm{H}$ para el conjunto de España con el P90 como grupo minoritario en las escuelas públicas es de 0,25 , mientras que para las privadas es de 0,13 .

Las diferencias entre CCAA son muy llamativas. De nuevo encontramos que, en la mayoría de las comunidades y para este $10 \%$ de los estudiantes con familias con mayor nivel socioeconómico y cultural, el subsistema público segrega más que el privado. Destacan muy claramente Canarias, donde la segregación en los centros públicos es de 0,25 , mientras que en los privados se queda en 0,12 . También existe esa diferencia marcada en Castilla-La Mancha $(0,17$ y 0,05), la Comunidad de Madrid $(0,25$ y 0,16), el País Vasco $(0,24$ y 0,15) o Navarra $(0,18$ y 0,10). En el Principado de Asturias, Cantabria, Cataluña, La Rioja y Aragón y Andalucía, por el contrario, segregan más los centros privados que los públicos (gráfico 5).

La descomposición de la segregación total de cada comunidad entre el $\mathrm{H}$ intrasubsistemas y el intersubsistemas (tabla 8) muestra que la segregación escolar por nivel socioeconómico del 10\% de los estudiantes de mayor nivel socioeconómico en el total estatal y en la gran mayoría de las comunidades se debe esencialmente a los centros públicos. Esto se observa muy especialmente en Canarias (con 0,10 puntos de diferencia) y Castilla-La Mancha (con 0,10). 
Gráfico 5. Segregación socioeconómica, en valores absolutos, de los centros educativos públicos y privados de Educación Secundaria Obligatoria en España. Índice de raíz cuadrada para el $10 \%$ de los estudiantes con familias con mayor nivel socioeconómico y cultural (P90) como grupo minoritario, por comunidades autónomas

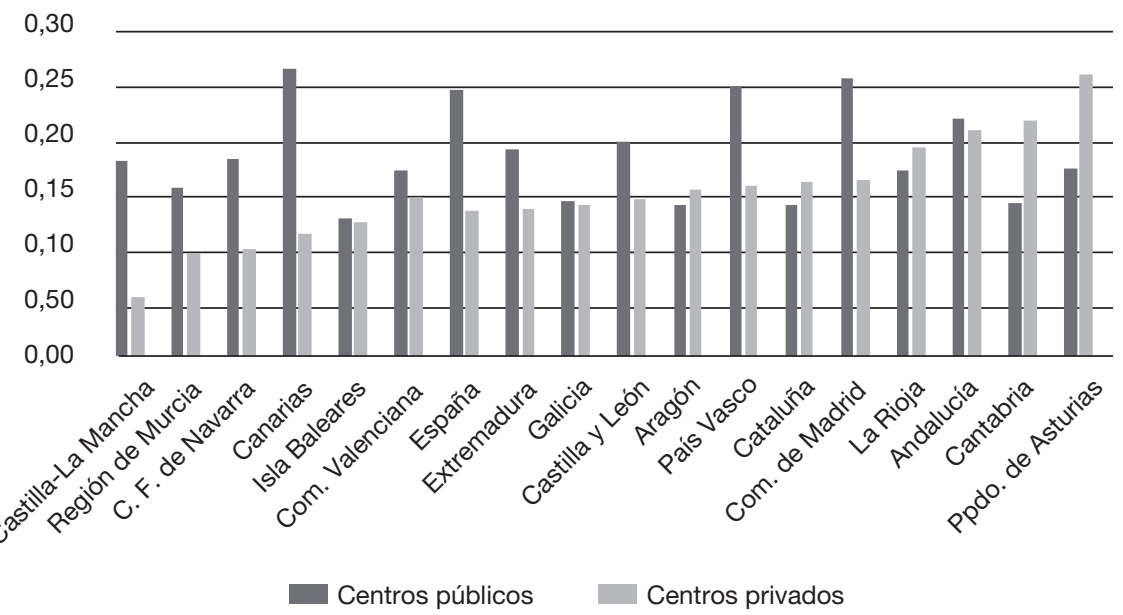

Nota: comunidades ordenadas por su índice de segregación en centros educativos privados.

Fuente: elaboración propia a partir de los datos de PISA-2015.

Tabla 8. Segregación en centros educativos públicos y privados en España. Descomposición del índice de raíz cuadrada para el $10 \%$ de los estudiantes con familias con mayor nivel socioeconómico y cultural (P90) como grupo minoritario, por comunidad autónoma

\begin{tabular}{|c|c|c|c|c|c|c|c|c|}
\hline & \multirow[b]{3}{*}{$\mathrm{H}$ total } & \multicolumn{6}{|c|}{$\mathrm{H}$ intrasubsistemas } & \multirow{3}{*}{$\begin{array}{c}\mathrm{H} \text { inter- } \\
\text { subsiste-mas }\end{array}$} \\
\hline & & \multicolumn{3}{|c|}{ Centros ed. públicos } & \multicolumn{3}{|c|}{ Centros ed. Privados } & \\
\hline & & $\mathrm{H}$ bruto & Pond. & Aport. & $\mathrm{H}$ bruto & Pond. & Aport. & \\
\hline España & 0,2458 & 0,2403 & 0,5118 & 0,1230 & 0,1312 & 0,4206 & 0,0552 & 0,0676 \\
\hline Andalucía & 0,2430 & 0,2141 & 0,6134 & 0,1313 & 0,2045 & 0,3316 & 0,0678 & 0,0438 \\
\hline Aragón & 0,1584 & 0,1365 & 0,5310 & 0,0725 & 0,1510 & 0,4140 & 0,0625 & 0,0234 \\
\hline Ppdo. de Asturias & 0,2480 & 0,1696 & 0,5007 & 0,0849 & 0,2553 & 0,4163 & 0,1063 & 0,0568 \\
\hline Islas Baleares & 0,1343 & 0,1251 & 0,5699 & 0,0713 & 0,1217 & 0,4181 & 0,0509 & 0,0120 \\
\hline Canarias & 0,2993 & 0,2596 & 0,5350 & 0,1389 & 0,1107 & 0,3253 & 0,0360 & 0,1244 \\
\hline Cantabria & 0,1956 & 0,1392 & 0,5668 & 0,0789 & 0,2124 & 0,3724 & 0,0791 & 0,0376 \\
\hline Castilla y León & 0,2145 & 0,1947 & 0,5014 & 0,0976 & 0,1427 & 0,3959 & 0,0565 & 0,0604 \\
\hline Castilla-La Mancha & 0,2097 & 0,1759 & 0,6294 & 0,1107 & 0,0527 & 0,2867 & 0,0151 & 0,0839 \\
\hline Cataluña & 0,2063 & 0,1371 & 0,4317 & 0,0592 & 0,1569 & 0,4996 & 0,0784 & 0,0687 \\
\hline Extremadura & 0,2092 & 0,1865 & 0,6060 & 0,1130 & 0,1331 & 0,3434 & 0,0457 & 0,0506 \\
\hline Galicia & 0,1677 & 0,1405 & 0,5952 & 0,0836 & 0,1359 & 0,3436 & 0,0467 & 0,0374 \\
\hline La Rioja & 0,1810 & 0,1683 & 0,4789 & 0,0806 & 0,1879 & 0,4184 & 0,0786 & 0,0217 \\
\hline Com. de Madrid & 0,2325 & 0,2513 & 0,4696 & 0,1180 & 0,1586 & 0,4942 & 0,0784 & 0,0362 \\
\hline Región de Murcia & 0,1591 & 0,1529 & 0,6699 & 0,1024 & 0,0938 & 0,3018 & 0,0283 & 0,0283 \\
\hline C. F. de Navarra & 0,1743 & 0,2069 & 0,4723 & 0,0977 & 0,1270 & 0,4644 & 0,0590 & 0,0502 \\
\hline País Vasco & 0,2086 & 0,3141 & 0,3056 & 0,0960 & 0,1402 & 0,4231 & 0,0593 & 0,0490 \\
\hline Com. Valenciana & 0,1767 & 0,1686 & 0,5557 & 0,0937 & 0,1429 & 0,4109 & 0,0587 & 0,0494 \\
\hline
\end{tabular}

Fuente: elaboración propia a partir de los datos de PISA-2015. 
Gráfico 6. Segregación escolar por nivel socioeconómico del subsistema de educación pública en educación secundaria en España para diferentes colectivos. Índice de raíz cuadrada para el P10, Q1, Q4 y P90 en NSEC como grupos minoritarios, por comunidad autónoma

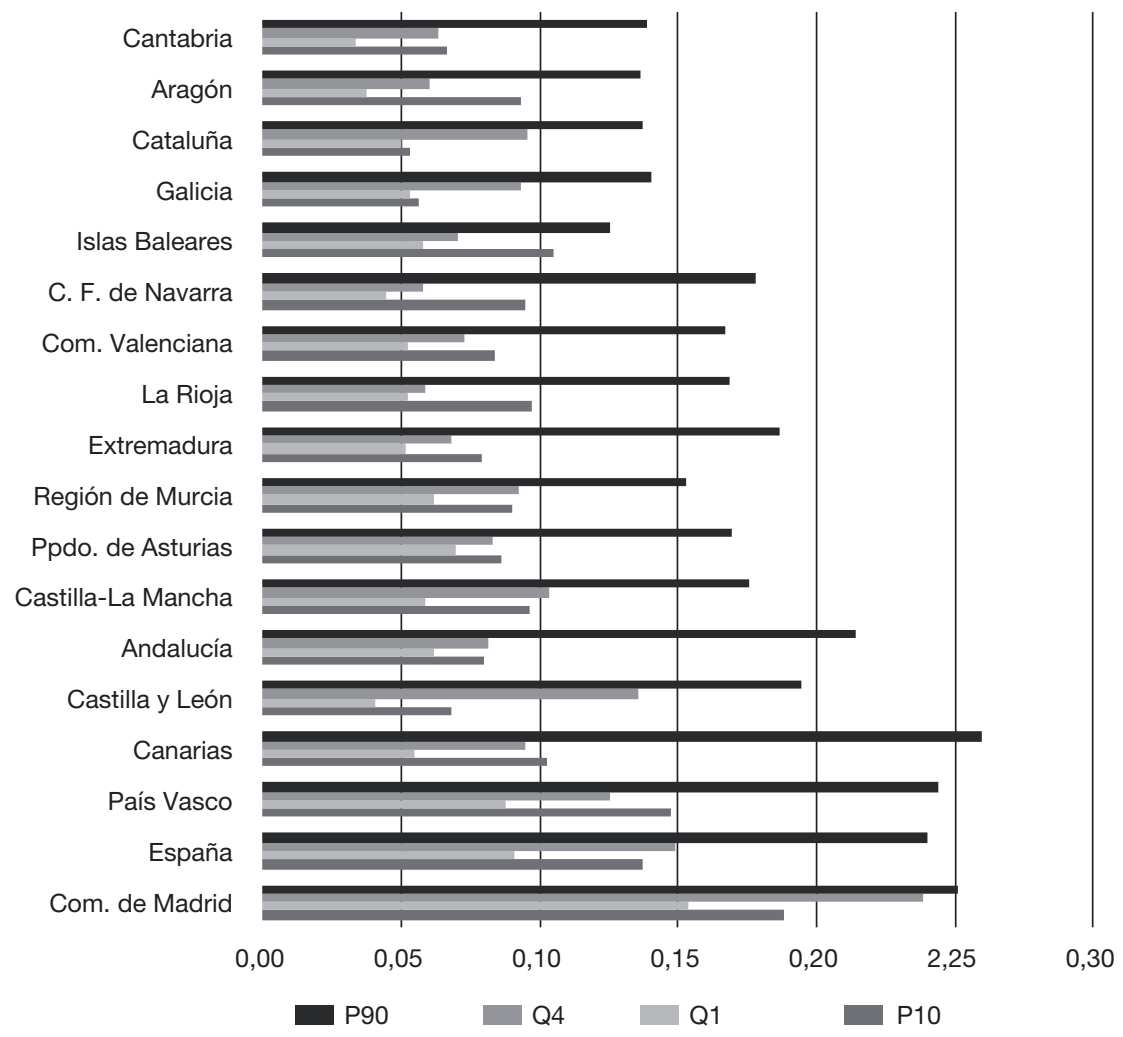

Nota: comunidades ordenadas por el promedio de los índices de segregación.

Fuente: elaboración propia a partir de los datos de PISA-2015.

En Asturias, Cataluña y Cantabria, por el contrario, los centros privados aportan más que los públicos a la segregación global.

Una panorámica de la segregación bruta de los subsistemas públicos y privados para cada uno de los cuatro colectivos estudiados nos ofrece una interesante visión de los diferentes comportamientos de cada comunidad.

El análisis de la segregación del subsistema de educación pública (gráfico 6) muestra que en todas las comunidades la segregación es mayor para el $10 \%$ de los estudiantes con familias con un mayor nivel socioeconómico y cultural (P90), lo que implica que estos estudiantes no se escolarizan en centros públicos.

También aquí existen grandes diferencias entre las comunidades autónomas. Los extremos son las dos comunidades insulares: Canarias y las Islas 
Gráfico 7. Segregación escolar por nivel socioeconómico del subsistema de educación privada en educación secundaria en España para diferentes colectivos. Índice de raíz cuadrada para el P10, Q1, Q4 y P90 en NSEC como grupos minoritarios, por comunidad autónoma

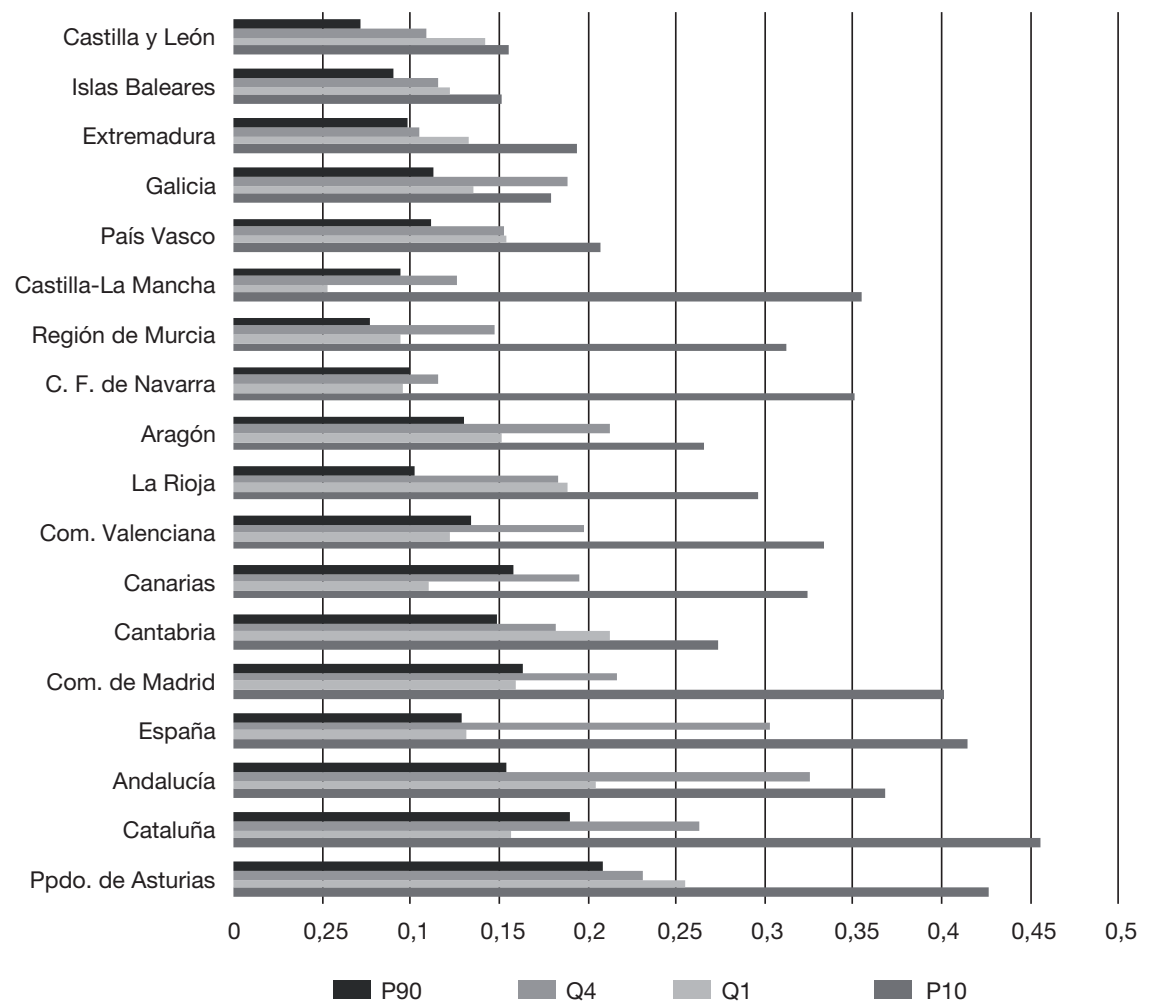

Nota: comunidades ordenadas por el promedio de los índices de segregación.

Fuente: elaboración propia a partir de los datos de PISA-2015.

Baleares. En el primer caso, la magnitud de la segregación escolar es muy alta para el P90, es decir para los estudiantes con familias con mayor nivel socioeconómico y cultural, pero menor para el resto de los colectivos. En las Islas Baleares, sin embargo, hay pocas diferencias en la segregación en función del colectivo implicado.

Para el subsistema de educación privada, el comportamiento es justo el contrario. La segregación se da entre los estudiantes con familias con menor nivel socioeconómico y cultural (tanto P10 como Q1), aunque con comportamientos disímiles entre comunidades. Mientras que en Cataluña hay grandes diferencias en la magnitud de la segregación en función del colectivo, en Galicia o las Islas Baleares las diferencias son muy pequeñas. 


\section{Conclusiones}

La segregación escolar por nivel socioeconómico es un problema real y complejo para España. La presente investigación ha dejado al descubierto las situaciones generadas por políticas educativas diferentes en cada comunidad. Los resultados muestran que no existe una tendencia homogénea para todo el Estado y la cuestión adquiere una u otra característica según la comunidad autónoma de la que se trate. Con ello, se visibiliza el impacto, tanto positivo como negativo, que las políticas de los gobiernos regionales tienen sobre la segregación escolar, pues la magnitud del problema y el modo en que afecta a los distintos colectivos presentan una gran variabilidad entre autonomías. Las situaciones varían desde una alta segregación en la Comunidad de Madrid, Canarias o Cataluña, generada por los centros públicos hacia los estudiantes de familias con más recursos — en los dos primeros casos_, o por los centros privados hacia los estudiantes con familias de menos recursos - en el tercer caso-; hasta una baja segregación global, como en el caso de las Islas Baleares, donde existen bajas diferencias en la segregación de centros públicos y privados.

Tras considerar la segregación por grupo minoritario, los resultados de la investigación indican, en primer lugar, que se trata de un fenómeno que afecta tanto a los más favorecidos como a los menos. También aquí el mapa nacional demostró ser complejo, basta retomar el ejemplo de las comunidades autónomas más afectadas para encontrar que en la Comunidad de Madrid, por ejemplo, el índice de segregación es más alto para los estudiantes de familias con mayor nivel socioeconómico (P90), mientras que en Cataluña afecta más al 10\% de estudiantes cuyas familias tienen menor nivel socioeconómico (P10). Sin embargo, existe una tendencia general para el conjunto de España y para la mayoría de las comunidades que demuestra la puntuación más alta en el nivel de segregación para el 10\% de estudiantes cuyos padres tienen mayor NSEC; seguido del $10 \%$ de los que menos tienen, del $25 \%$ de los que menos y del $25 \%$ de los que más. Estos resultados indican que la segregación es un problema para la sociedad en su conjunto ya que, en términos de integración social, el caso es igual de preocupante respecto a uno u otro grupo de estudiantes.

En segundo lugar, se encuentra que centros públicos y privados inciden de manera distinta en el fenómeno de la segregación. La distribución de la educación pública y privada es diferente para cada comunidad y para cada colectivo, pero aun así pueden realizarse algunos comentarios generales que ayudan a entender la relación entre la titularidad de las escuelas y la segregación escolar. Para el 10\% de estudiantes con menor NSEC, las escuelas privadas presentan una gran variabilidad en su índice de segregación, existiendo regiones en las que alcanza un índice muy alto (Cataluña, 0,46; Principado de Asturias, 0,43, y Comunidad de Madrid, 0,40). Para el 10\% de estudiantes con mayor NSEC, en cambio, los centros públicos alcanzan índices altos, pero sin llegar a esos extremos (Canarias, 0,26 y Comunidad de Madrid, 0,25).

Los resultados del estudio indican que la segregación de los centros privados para el Q1 y, especialmente, para el P10 es considerablemente mayor 
que la de los públicos. Aunque las cifras varían para todas las comunidades, se confirma que la educación privada segrega a los estudiantes más desfavorecidos. Respecto a los estudiantes con mayor NSEC, la segregación oscila ampliamente para el Q4 según titularidad del centro y comunidad autónoma, pero para el P90 el sistema público segrega en mayor medida. Este es un indicador de que los estudiantes de mayor nivel socioeconómico no se escolarizan en centros públicos.

No es fácil buscar una explicación tentativa que nos ayude a entender las importantes divergencias encontradas en la segregación entre las diferentes comunidades autónomas y su comportamiento diferencial según la titularidad del centro. En todo caso, nos invita a pensar que las políticas educativas públicas juegan un papel determinante en la segregación escolar. Podemos apuntar alguna hipótesis de los casos más extremos, la gran segregación de la Comunidad de Madrid en los centros públicos y la de Cataluña en los privados. La Comunidad de Madrid ha iniciado en los últimos años una política neoliberal de generación de un sistema educativo de cuasimercado escolar, fomentando la libre elección de centros y la competencia entre ellos. Algunas medidas tomadas son la existencia del distrito único, la publicación de rankings de centros de forma más o menos velada o el sistema de centros bilingües, que desplaza a los estudiantes más vulnerables. Cataluña, por su parte, es la comunidad donde más claramente la escuela privada ha escolarizado a los estudiantes de familias de mayor nivel socioeconómico, relegando a los de menor nivel a los centros públicos (como vimos en la tabla 4), lo que ayuda a explicar esa segregación tan alta.

La investigación ha encontrado evidencias sobre el efecto segregador de la educación privada (concertada y no concertada) en España en Educación Secundaria Obligatoria, pues tiende a sostener y fortalecer la distancia entre los estudiantes con familias de niveles socioeconómicos y culturales extremos. Los centros privados atraen a los hijos e hijas de las familias con mayor nivel socioeconómico, lo que influye en la segregación de los estudiantes pertenecientes a familias con menor nivel. Además, el escaso porcentaje de centros privados no concertados de educación secundaria (3,58\% para el total del Estado) deja al descubierto que el problema debe analizarse sobre la base de la normativa que rige el financiamiento público de los centros. En este sentido, la gravedad de la segregación en la educación privada es doble. Por un lado, refleja y profundiza las desigualdades socioeconómicas y culturales de los estudiantes; por el otro, deja al trasluz el incumplimiento de la normativa bajo la cual los centros concertados reciben financiamiento público, especialmente en lo que atañe a la igualdad en el acceso a la educación y la libertad de elección de centro.

Mientras tanto, los centros públicos son la opción de los que no pueden elegir. Los resultados indican que la probabilidad de estudiar en un centro público aumenta cuanto menor es el nivel socioeconómico y cultural de la familia del estudiante y estos resultados llaman a la reflexión en, al menos, dos puntos importantes. Por un lado, exige cotejar este resultado con la legislación vigente. 
Como se ha visto, el artículo 84 de la LOMCE establece el marco normativo que rige los centros financiados con fondos públicos (públicos y concertados) y mediante el cual se garantiza el acceso en condiciones de igualdad y la libertad de elección de centro. Estas prerrogativas se cumplen en el caso de los estudiantes pertenecientes a familias con mayor nivel socioeconómico y cultural, que tienden a elegir los mismos centros privados para su escolarización; pero no en el caso de los estudiantes de familias menos favorecidas.

Por otro lado, el problema de la segregación en relación con la titularidad del centro es un problema también en lo que refiere a la desvalorización de la educación pública. La educación pública parece estar ligada a la imposibilidad de elegir, puesto que, en tanto aumenta la posibilidad económica de las familias para elegir centro, estas tienden a escoger centros privados. De esta manera la educación pública tiende a asociarse con una opción menor y es producto de un descuido y una desatención no casual. El riesgo radica en que esta asociación desestime la posibilidad de que la educación pública pueda ser valorada también por criterios de excelencia, tal como sugieren algunas de las investigaciones citadas al comienzo.

Finalmente, es preciso especificar algunas cuestiones metodológicas respecto a la investigación, pues trabajar con datos procedentes de una evaluación internacional como PISA-2015 tiene muchas ventajas, pero también algunas limitaciones. Entre las ventajas se encuentra la posibilidad de acceder a datos de gran actualidad, a una muestra de gran tamaño, estadísticamente representativa de cada comunidad autónoma, así como a una medición muy fiable del nivel socioeconómico y cultural de las familias de los estudiantes. Las limitaciones, en cambio, proceden de las fuentes. Por una parte, contar con datos únicamente de un aula por centro educativo puede producir sesgos como, por ejemplo, aquellos debidos a la segregación intraescolar. Otro limitante reside en no contar con datos desagregados de centros privados concertados y no concertados. Sin embargo, teniendo en cuenta que, como se señaló en el apartado metodológico, el porcentaje de estudiantes matriculados en centros privados es muy pequeño (del 3,53\% para el conjunto del Estado), no sería muy arriesgado generalizar estos resultados a los centros concertados. Faltaría, pues, un estudio de los centros no concertados.

Aun así, los hallazgos de la investigación exigen una seria consideración. Sugieren que hablar de segregación escolar en España no es referirse solo a la segregación de los menos favorecidos, puesto que se ha demostrado que es mayor la segregación de los que tienen un nivel socioeconómico y cultural más alto. Los resultados han puesto en evidencia la progresiva pérdida de integración social, la desvalorización de la escuela pública y la incapacidad del sistema educativo de garantizar el cumplimiento de una ley que proclama el acceso a la educación en condiciones de igualdad y la libertad de elección de centro. En pocas palabras, la investigación llama la atención sobre un sistema educativo que no cumple a la hora de garantizar la igualdad de oportunidades que proclama y demuestra que es imprescindible una acción política que revierta las tendencias a la segregación escolar. 


\section{Referencias bibliográficas}

ABburrà, L. (2005). As Good as the Others. Northern Italian Students and their Peers in other European Regions. Piamonte: Istituto Ricerche Economico Sociali del Piemonte.

Alegre Canosa, M. Á. (2010). «Casi-mercados, segregación escolar y desigualdad educativa: una trilogía con final abierto». Educação \& Sociedade, 31 (113), 1157 1178. <https://doi.org/10.1590/s0101-73302010000400006>.

Allen, R. y VignOles, A. (2007). "What Should an Index of School Segregation Measure?». Oxford Review of Education, 33 (5), 643-668. <https://doi.org/10.1080/03054980701366306>.

Angrist, J.; Bettinger, E.; Bloom, E.; King, E. y Kremer, M. (2002). «Vouchers for Private Schooling in Colombia: Evidence from a Randomized Natural Experiment». The American Economic Review, 92 (5), 1535-1558. <https://doi.org/10.3386/w8343>.

Arcidiácono, M.; Cruces, G.; Gasparini, L.; Jaume, D.; Serio, M. y VázQuez, E. (2014). La segregación escolar público-privada en América Latina. Santiago de Chile: CEPAL.

Barbetta, G. P. y Turati, G. (2003). «Efficiency of Junior High Schools and the Role of Proprietary Structure». Annals of Public and Cooperative Economics, 74 (4), 529-552. <https://doi.org/10.1111/j.1467-8292.2003.00234.x>.

Benito, R.; Alegre, M. A. y GonZÁlez-Balletbò, I. (2014). «School Segregation and its Effects on Educational Equality and Efficiency in 16 OECD Comprehensive School Systems». Comparative Education Review, 58 (1), 104-134. $<$ https://doi.org/10.1086/672011>.

Benito, R. y GonZÁlez, I. (2007). Processos de segregació escolar a Catalunya. Barcelona: Editorial Mediterrània. <https:/www.fbofill.cat/sites/default/files/464.pdf>.

BetTs, J. y FAIRliE, R. (2014). «Explaining Ethnic, Racial, and Immigrant Differences in Private School Attendance». Journal of Urban Economics, 22 (5), 25-46. $<$ https://doi.org/10.1006/juec.2000.2207>.

BONAL, X. (2002). «El balance público-privado en el sistema de enseñanza español». Educar, 29, 11-29. $<$ https://doi.org/10.5565/rev/educar.323>

- (2012). «Education Policy and School Segregation of Migrant Students in Catalonia: The Politics of Non-Decision-Making». Journal of Education Policy, 27 (3), 401-421. <https://doi.org/10.1080/02680939.2011.645168>.

Coleman, J. S. y Hoffer, T. (1987). Public and Private High Schools: The Impact of Communities. Nueva York: Basic Books.

Coleman, J. S.; Hoffer, T. y Kilgore, S. (1982). High School Achievement: Public, Catholic, and Private Schools Compared. Nueva York: Basic Books.

Coleman, J. S.; Kelly S. D. y Moore, J. A. (1975). Trends in School Segregation, 1968-73. Washington, DC: The Urban Institute.

Corten, R. y Dronkers, J. (2006). «School Achievement of Pupils from the Lower Strata in Public, Private Government-Dependent and Private Government-Independent Schools: A Cross-National Test of the Coleman-Hoffer Thesis». Educational Research and Evaluation, 12 (2), 179-208. $<$ https://doi.org/10.1080/13803610600587032>. 
Dronkers, J. (2004). «Do Public and Religious Schools Really Differ? Assessing the European Evidence». En: Wolf, P. J. y MACEDO, S. (ed.). Educating Citizens. International Perspectives on Civic Values and School Choice, (287-314). Washington, D C: Brookings Institution Press. <https://doi.org/10.5860/choice.42-6620>.

Dronkers, J. y RoberT, P. (2008). «Differences in Scholastic Achievement of Public, Private Government-Dependent, and Private Independent Schools a Cross-National Analysis». Educational Policy, 22 (4), 541-577. <https://doi.org/10.1177/0895904807307065>.

Elacqua, G.; Martínez, M.; Santos, H. y Urbina, D. (2016). «Tensiones públicoprivado y el diseño de los sistemas educativos: ¿Qué nos dice PISA?». Pro-Posições, 23 (2), 105-123. <https://doi.org/10.1590/s0103-73072012000200008>.

Eurostat (2016). Eurostat. Education and Training Database. $<$ http://ec.europa.eu/eurostat/web/education-and-training/data/database>.

FERNÁNDEZ ENGUITA, M. (2008). «Escuela pública y privada en España: La segregación rampante». Revista de la Asociación de Sociología de la Educación, 1 (2), 42-69.

Fertig, M. (2003). "Who's to Blame? The Determinants of German Students' Achievement in the PISA 2000 Study». IZA Discussion Paper Series, 739. $<$ https://ssrn.com/abstract $=392040>$

FRANKEL, D. y O. VOLIJ (2011). Measuring school segregation. Journal of Economic Theory, 146(1), 1-38. https://doi.org/10.1016/j.jet.2010.10.008>.

García Castaño, F.J. y Rubio Gómez, M. (2013). "Juntos pero no revueltos: Procesos de concentración escolar del alumnado extranjero en determinados centros educativos». Revista de Dialectología y Tradiciones Populares, 68(1), 7-31. https://doi.org/10.3989/rdtp.2013.01.001>.

GARCÍA FERnÁNDEZ, J. A. y MORENO, I. (2002). La respuesta a las necesidades educativas de los hijos de inmigrantes en la Comunidad de Madrid. Madrid: CES de Madrid.

Goldhaber, D. D. (1996). "Public and Private High Schools: Is School Choice an Answer to the Productivity Problem?». Economics of Education Review, 15 (2), 93-109. <https://doi.org/10.1016/0272-7757(95)00042-9>.

Gorard, S. (2000). Education and Social Justice. Cardiff: University of Wales Press.

- (2009). "Does the Index of Segregation Matter? The Composition of Secondary Schools in England since 1996». British Educational Research Journal, 35 (4), 639-652. <https://doi.org/10.1080/01411920802642389>.

Gorard, S. y SMITH, E. (2004). "An International Comparison of Equity in Education Systems». Comparative Education, 40 (1), 15-28. <https://doi.org/10.1080/0305006042000184863>.

Gorard, S. y TAYLOR, C. (2002). "What is Segregation? A Comparison of Measures in Terms of 'Strong' and 'Weak' Compositional Invariance». Sociology, 36 (4), $875-895$. <https://doi.org/10.1177/003803850203600405>.

Hutchens, R. M. (2001). «Numerical Measures of Segregation: Desirable Properties and their Implications». Mathematical Social Sciences, 42, 13-29.

- (2004). "One Measure of Segregation». International Economic Review, 45 (2), 555-578. <https://doi.org/10.1111/j.1468-2354.2004.00136.x>. 
JEnkins, S. P.; Micklewright, J. y Schnepf, S. V. (2008). «Social Segregation in Secondary Schools: How does England Compare with other Countries?». Oxford Review of Education, 34 (1), 21-37. <https://doi.org/10.1080/03054980701542039>.

Mancebón-Torrubia, M. J. y Pérez-Ximénez de Embún, D. (2008). «El alumnado de los centros educativos aragoneses de enseñanza secundaria: segregación socioeconómica y académica por tipo de centro y percepciones de la calidad educativa». Economía Aragonesa, 35, 115-136.

- (2010). «Una valoración del grado de segregación socioeconómica existente en el sistema educativo español. Un análisis por comunidades autónomas a partir de PISA 2006». Regional and Sectoral Economic Studies, 10 (3), 129-148.

Mancebón-Torrubia, M. J.; Pérez-Ximénez de Embún, D.; Mediavilla, M. y GómeZ-SANCHO, J. M. (2015). Does Educational Management Model Matter? New Evidence for Spain by a Quasiexperimental Approach. Barcelona: IEB.

Morgan, S. L. y Sørensen, A. B. (1999). «Parental Networks, Social Closure, and Mathematics Learning: a Test of Coleman's Social Capital Explanation of School Effects». American Sociological Review, 64, 661-681. <https://doi.org/10.2307/2657368>.

Murillo, F. J. (2016). «Midiendo la segregación escolar en América Latina. Un análisis metodológico utilizando el TERCE». REICE. Revista Iberoamericana sobre Calidad, Eficacia y Cambio en Educación, 14 (4), 33-60. <https://doi.org/10.15366/reice2016.14.4.002>.

OCDE (2009). Diez pasos hacia la equidad en educación. París: OCDE.

- (2013). Equidad y calidad de la educación: ¿Cómo apoyar a estudiantes y escuelas en desventaja? París: OCDE.

- (2016). Programme of International Student Assessment (PISA 2015). París: OCDE.

Orfield, G. y LeE, C. M. (2005). Why Segregation Matters: Poverty and Educational Inequality. Boston, MA: Harvard University Press.

PORTES, A. (1998). «Social Capital: Its Origins and Applications in Modern Sociology». Annual Review of Sociology 24 (1), 1-24. <https://doi.org/10.1016/b978-0-7506-7222-1.50006-4>.

Poveda, D.; Franzé, A.; Jociles, M. I.; Rivas, A. M.; Villaamil, F.; Peláez, C. y SÁNCHEZ, P. (2007). «La segregación étnica en la educación secundaria de la ciudad de Madrid: un mapa y una lectura crítica». EMIGRA Working Papers, 91. <https:// ddd.uab.cat/pub/emigrawp/emigrawp_a2007n91/emigrawp_a2007n91p1.pdf>.

Reardon, S. F. y Owens, A. (2014). «60 Years after Brown: Trends and Consequences of School Segregation». Annual Review of Sociology, 40, 199-218. <https://doi.org/10.1146/annurev-soc-071913-043152>.

Rivkin, S. G. (2000). «School Desegregation, Academic Attainment, and Earnings». Journal of Human Resources, 35 (2), 333-346. $<$ https://doi.org/10.2307/146328>.

Rumberger, R. y Palardy, G. (2005). «Does Segregation still Matter? The Impact of Student Composition on Academic Achievement in High School». The Teachers College Record, 107 (9), 1999-2045.

SAN SEgUndo, M. J. (1991). Evaluación del sistema educativo a partir de datos individuales. Madrid: Universidad Carlos III de Madrid.

Sandefur, R. L. y Laumann, E. O. (1998). "A Paradigm for Social Capital». Rationality and Society, 10 (4), 481-501. <https://doi.org/10.1016/b978-0-7506-7222-1.50007-6>. 
SíndiC De Greuges (2008). La segregación escolar en Cataluña. Barcelona: Síndic de Greuges de Catalunya.

- (2016). La segregación escolar en Cataluña (I): La gestión del Proceso de Admisión del Alumnado. Barcelona: Síndic de Greuges de Catalunya.

Smith, J. y NAYLOR, R. A. (2005). «Schooling Effects on Subsequent University Performance: Evidence for the UK University Population». Economics of Education Review, 24, 549-562. <https://doi.org/10.1016/j.econedurev.2004.07.016>

Stephan, W. (2013). School Desegregation: Past, Present, and Future. Nueva York: Springer.

Stevans, L. K. y Sessions, D. N. (2000). «Private/Public School Choice and Student Performance Revisited». Education Economics, 8 (2), 169-184. <https://doi.org/10.1080/096452900410730>.

Tokman, A. (2002). Is Private Education Better? Evidence from Chile. Santiago: Banco Central de Chile.

UNESCO (2005). Segundo Estudio Regional Comparativo y Explicativo (SERCE). Santiago: UNESCO/LLECE.

WiLsON, W. J. (1987). The Truly Disadvantaged. Chicago, IL: University of Chicago Press. 